\title{
THE NEUROSCIENCE OF GROOVE: NEURAL MECHANISMS MARRYING MUSIC AND MOVEMENT \\ by
}

Gabriel A. Nespoli

Master of Arts, Ryerson University, 2014

Bachelor of Science, McGill University, 2007

\author{
A dissertation \\ presented to Ryerson University \\ in partial fulfillment of the \\ requirements for the degree of \\ Doctor of Philosophy \\ in the program of \\ Psychology
}

Toronto, Ontario, Canada, 2018

(C) Gabriel A. Nespoli 2018 


\section{AUTHOR'S DECLARATION FOR ELECTRONIC SUBMISSION OF A DIS- SERTATION}

I hereby declare that I am the sole author of this dissertation. This is a true copy of the dissertation, including any required final revisions, as accepted by my examiners.

I authorize Ryerson University to lend this dissertation to other institutions or individuals for the purpose of scholarly research.

I further authorize Ryerson University to reproduce this dissertation by photocopying or by other means, in total or in part, at the request of other institutions or individuals for the purpose of scholarly research.

I understand that my dissertation may be made electronically available to the public. 


\begin{abstract}
The Neuroscience of Groove: Neural Mechanisms Marrying Music and Movement
\end{abstract}

Doctor of Philosophy, 2018

Gabriel A. Nespoli

Psychology, Ryerson University

Music has a long history of being associated with movement synchronization such as foot-tapping or dance. These behaviours are easier with some music compared to others, and the reasons for this are not well understood. Groove is a quality of music that compels synchronous movement in the listener, and certain acoustic and musical features have been identified that contribute to a sense of groove. Neurons have been found to entrain to the beat of music. Combining these two ideas, it is reasonable to predict that neural populations involved in movement (i.e., premotor areas) would entrain more to high-groove than to low-groove music. This dissertation explores some of the psychological, musical, and acoustic aspects of music that contribute to neural entrainment in premotor areas of the brain. Study 1 investigates the effects of feelings of groove on premotor entrainment, using stimuli that have been rated on extent of groove in a previous study. Study 2 investigates the musical feature of syncopation - which has previously been found to be associated with sense of groove - on extent of premotor entrainment and behavioural synchronization ability. Study 3 investigates the effects of acoustic features that have been found to be related to 
groove and movement synchronization such as event density and percussiveness. The pattern of results across all studies suggests that the complexity of the rhythms in the stimulus determines the extent of beat entrainment. Feelings of groove, however, are better characterized by "beat complexity", which depends on a) the extent to which the listener perceives the beat, and b) the extent to which other rhythmic elements of the music compete with the beat. A network of brain areas integral to the perception of groove is proposed, where activation of premotor areas enables music to drive motor output. 


\section{Acknowledgements}

I would like to thank my advisor, Frank Russo, who has been a great friend, colleague, and mentor for many years. A lot of life has happened in the decade since I became a member of the lab, and I have always appreciated your understanding, support, and advice. You have created something really special in the SMART Lab, and I will forever cherish my time there. Thanks to everybody who has spent time there for creating such a wonderful atmosphere.

Thanks to Alex Andrews and to Carson Pun for our many conversations about signal processing, coding, and kids. Thanks to Luke Reid for putting up with all my music talk, and for encouraging me to pursue this in the first place. Thanks to Paolo Ammirante and Sean Gilmore for your thoughtful comments and insights. Thanks to Meg Moulson, Todd Girard, and Lixia Yang for your comments on my various long documents. And a big thanks to everyone in the Psychology Department, who have made my time at Ryerson a special one. This place is full of truly wonderful people.

Mom and Pop, Karen and Cy, thank you for all that you do, but especially for your help with the kids. Olly and June, thanks for helping me keep things in perspective, even if you did not know you were doing it, or if it was the best time to deliver it. Last but foremost to Erin, for your constant love and endless patience. You are a beautiful person, partner, and mother. I cannot wait for what happens next. 
For Erin, Olly, and June. 
Abstract

List of Tables $\quad$ ix

List of Figures $\quad$ X

List of Appendices $\quad$ xi

Abbreviations $\quad$ xii

The Neuroscience of Groove: Neural Mechanisms Marrying $\begin{array}{ll}\text { Music and Movement } & 1\end{array}$

The Feeling of Groove . . . . . . . . . . . . . . . . . . . . . . . . . 2

Rhythm, Syncopation, and Groove . . . . . . . . . . . . . . . 4

Acoustic Properties of Groove . . . . . . . . . . . . . . . . . . 5

Neural Entrainment to Music . . . . . . . . . . . . . . . . . . 7

Neural Mechanisms . . . . . . . . . . . . . . . . . . . . . 14

Research Aims . . . . . . . . . . . . . . . . . . . 17

Study 1: Psychological Aspects of Music (Feelings of Groove) 18

Methods . . . . . . . . . . . . . . . . . . 20

Results . . . . . . . . . . . . . . . . . . . 24

Discussion . . . . . . . . . . . . . . . . . . . . . 25

Conclusions . . . . . . . . . . . . . . . . . . . . . . 28

Study 2: Musical Aspects of Music (Syncopation) 29

Methods ... . . . . . . . . . . . . . . . . 32

Results . . . . . . . . . . . . . . . . . . . 38

Discussion . . . . . . . . . . . . . . . . . . 42

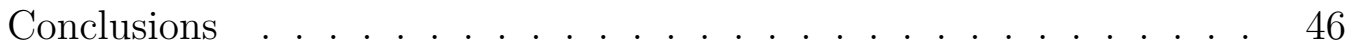

Study 3: Acoustic Aspects of Music $\quad 47$ 
Methods ........................ 50 50

Results ........................... 55

Discussion . . . . . . . . . . . . . . . . . . . . 59

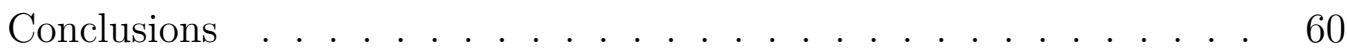

General Discussion $\quad 62$

Beat Complexity . . . . . . . . . . . . . . . . 63

Endogenous Oscillations . . . . . . . . . . . . . . 66

The Groove Pathway . . . . . . . . . . . . . . . . . . 67

Limitations and Future Directions . . . . . . . . . . . . . . 69

Conclusion . . . . . . . . . . . . . . . . . . 71

$\begin{array}{ll}\text { Appendices } & 72\end{array}$

$\begin{array}{ll}\text { References } & 78\end{array}$ 
List of Tables

1 List of stimuli (Study 2) . . . . . . . . . . . . . . . 34

2 Ratings model using acoustic features . . . . . . . . . . . 56

3 Ratings model applied to premotor entrainment to the beat. . . 56

4 Tapping variability model using acoustic features . . . . . . . . 57

5 Tapping model applied to PMC entrainment to the beat. . . . . 58

6 Premotor entrainment model using acoustic features . . . . . . 58

7 Beat complexity . . . . . . . . . . . . . . . . . . . . 64

$8 \quad$ List of stimuli (Study 1) . . . . . . . . . . . . . . . . 72

$9 \quad$ List of included data (Study 1) . . . . . . . . . . . . . . 73

10 Correlations of acoustic features for the ratings model. . . . . . 75

11 Correlations of acoustic features for the tapping model . . . . . 76

12 Correlation table of acoustic features and premotor entrainment to the beat. . . . . . . . . . . . . . . 76 


\section{List of Figures}

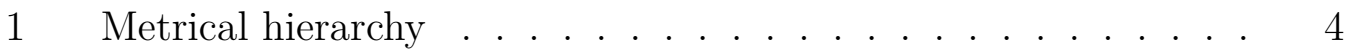

2 Steady-state evoked potentials . . . . . . . . . . . . . 8

3 Beta-band entrainment to a metronome . . . . . . . . . . . . 11

4 Neural entrainment to the beat of natural music (Study 1) . . . 25

$5 \quad$ Correlation between neural entrainment and tempo (Study 1) . 26

6 Talairach coordinates of selected ICs (Study 2) . . . . . . . . . 37

$7 \quad$ Ratings of wanting to move by syncopation degree (Study 2) . . 39

8 Tapping variability by syncopation degree (Study 2) . . . . . . 40

$9 \quad$ Neural entrainment by syncopation degree (Study 2) . . . . . . 41

10 Neural entrainment by metric level (Study 2) . . . . . . . . . . 42

11 Neural entrainment by syncopation degree and metric level (Study 2) . . . . . . . . . . . . . . . . . . . . . . 43

12 Talairach coordinates of selected ICs (Study 3) . . . . . . . . . 54

13 Event variability and PMC entrainment (Study 3) . . . . . . . 57

14 Percussiveness and PMC entrainment (Study 3) . . . . . . . . . 59 


\section{List of Appendices}

Appendix A ........................ . . . 72

Appendix B ....................... . . . 75 


\section{Abbreviations}

$\begin{array}{ll}\text { BA } & \text { Broadmann's Area } \\ \text { BG } & \text { Basal Ganglia } \\ \text { BOLD } & \text { Blood Oxygenation Level Dependent signal } \\ \text { EEG } & \text { Electro-encephalography } \\ \text { EMG } & \text { Electro-myography } \\ \text { IC } & \text { Independent Component } \\ \text { ICA } & \text { Independent Components Analysis } \\ \text { MEG } & \text { Magneto-encephalography } \\ \text { MEP } & \text { Motor-Evoked Potential } \\ \text { MRI } & \text { Magnetic Resonance Imaging } \\ \text { fMRI } & \text { Functional Magnetic Resonance Imaging } \\ \text { PMC } & \text { Pre-Motor Cortex } \\ \text { SMA } & \text { Supplementary Motor Area } \\ \text { SSEP } & \text { Steady-State Evoked Potential } \\ \text { TMS } & \text { Transcranial Magnetic Stimulation }\end{array}$


The Neuroscience of Groove: Neural Mechanisms Marrying

Music and Movement

Music has a long history that is richly associated with movement. Mothers sing to their infants with exaggerated facial expressions, members of a marching band synchronize their steps, and concert-goers coordinate dance movements with the music and one-another. Music can even compel movement, creating in a listener the desire to move along. This quality of music - groove - is one that makes music listening both enjoyable and motivating (Madison, 2006). Music becomes a catalyst for synchronized movement, effortlessly bringing people together in many ways. When mothers sing to their infants, they are coordinating voice and facial expressions in a manner that invites the infant to increase attention at specific moments in time. Marching songs can embolden an army, allowing individual soldiers to feel strength in numbers and advance headlong into battle. Enjoying a concert with others creates a sense of unity as everybody sways together in synchrony.

Music provides an excellent temporal framework for synchronizing movements (Repp, 2003). Several studies have investigated aspects of music that contribute to groove (Davies, Madison, Silva, \& Gouyon, 2013; Madison, Gouyon, Ullén, \& Hörnström, 2011; Sioros, Miron, Davies, Gouyon, \& Madison, 2014; Stupacher, Hove, \& Janata, 2016; Witek, Clarke, Wallentin, Kringelbach, \& Vuust, 2014) as well as its behavioural (Janata, Tomic, \& Haberman, 2012) and neural (Stupacher, Hove, Novembre, Schütz-Bosbach, \& Keller, 2013) consequences. Areas of the brain related to movement, such as premotor cortex (PMC), have been implicated in rhythm perception (Chen, Penhune, \& Zatorre, 2008; Fujioka, Trainor, Large, \& Ross, 2012; Grahn \& Brett, 2007), so it seems likely that PMC activation is somehow involved with feelings of groove. Thus, studying aspects of music that modulate PMC activity is of great importance for understanding how music can compel movement.

What is it about music that makes us want to move? Why is it sometimes difficult to resist the urge to move when you hear music? In this dissertation, 
I attempt to answer these questions by investigating the neural mechanisms involved in the perception of groove and the manner in which they are influenced by musical and psychoacoustic properties of music. Specifically, I describe a mechanism by which the patterns of notes and the temporal structure inherent in music can capitalize on neurons' ability to entrain to periodic stimuli. If neurons in motor-related brain areas become entrained, the motor system is primed for synchronizing movement with the musical stimulus. I propose that this synchronized motor activity is what elicits the desire to move. In three studies, I investigated different aspects of music that contribute to groove-psychological, musical, and acoustical — and how they might drive neural entrainment of motorrelated areas. First, I take a broad approach by investigating neural entrainment in a collection of music that varies in its extent of groove (as rated by participants in a study by Janata, Tomic, and Haberman (2012)). Second, I explore the musical feature of syncopation, and how these kinds of rhythms can contribute to neural entrainment. Last, I assess acoustic features of music in order to understand the optimal conditions for eliciting neural entrainment.

\section{The Feeling of Groove}

Colloquially, groove in music is when you cannot help but tap your foot along with what you are hearing. Groove feels like a gentle and continuous push forward, where the music grabs you and invites to pull you along. In a particularly eloquent description, Roholt (2014) likens groove to the feeling of driving a car on the freshly-packed grooves of snow left by rush-hour traffic after a heavy snowfall: "You have some sense of the firmness and path of these grooves...as you feel the car being pulled, pushed, and carried along" (p. 1). Madison (2006) defines groove more explicitly as "wanting to move some part of the body in relation to some aspect of the sound pattern" (p. 201). When asked to rate various statements about the definition of groove, participants consistently endorse statements containing words emphasizing movement and rhythm (e.g., move, dance, beat, rhythm) or compulsion (e.g., feel, make, want), such as "the groove depends on the extent to which the music makes you want to move" 
(Janata et al., 2012).

Music that is high in groove has been found to support behavioural synchronization, such as the ability to accurately tap along to the beat of the music. Janata et al. (2012) created a corpus of 148 musical excerpts (20 seconds in duration) and had participants rate each excerpt on "the extent to which the music 'grooves'" (p. 5). Based on these groove ratings they selected a subset of excerpts representing high-, mid-, and low-groove categories, and asked a separate group of participants to tap along with each excerpt. When participants were asked to tap along to the beat (i.e., isochronous ${ }^{1}$ tapping), their taps were more synchronized with the beat for high- than low-groove excerpts. Additionally, when participants were given freedom to tap as they liked (i.e., free-form tapping), the periodicities present in their tapping were the same as those present in the excerpt itself. This suggests that groove supports a person's ability to move along in synchrony with the music.

Janata et al. (2012) also video recorded participants during a no-tapping condition, where they were instructed to simply listen to the music. Blind coders rated these video-only recordings for spontaneous movement, which revealed more spontaneous movement while listening to high-groove music. This suggests that not only can groove support accurate behavioural synchronization, it can compel movement. Participants could not help but move along to highgroove music. Since these studies used natural music and not controlled auditory sequences as stimuli, the results could be due to a variety of aspects of the music, such as preference, syncopation, or presence of percussion. The following two sections review evidence for how syncopation and acoustic properties of music might be related to feelings of groove.

\footnotetext{
${ }^{1}$ An isochronous sequence is a rhythm consisting of notes that are equally-spaced in time, such as a metronome.
} 


\section{Rhythm, Syncopation, and Groove}

Music comprises notes of varying duration, creating temporal patterns called rhythmic groups (London, 2012). Meter refers to the specific temporal context within which rhythmic groups are produced and perceived. It consists of a main underlying pulse (the beat), and subdivisions and groupings of the beat. These subdivisions and groupings are conceptualized as nested levels of a metrical hierarchy (Lerdahl \& Jackendoff, 1983), as seen in Figure 1, (adapted from Large \& Kolen, 1994). Each level consists of a set of equally spaced beats, and levels are related to one another by simple integer ratios (typically 2, 3, 4, 6, or 8). Points in time where many beat levels align are considered points of metrical accent, and tend to co-occur with notes that are longer, louder, or more tonally stable. When people tap their foot to music they are typically synchronizing their taps with these metrical accents.

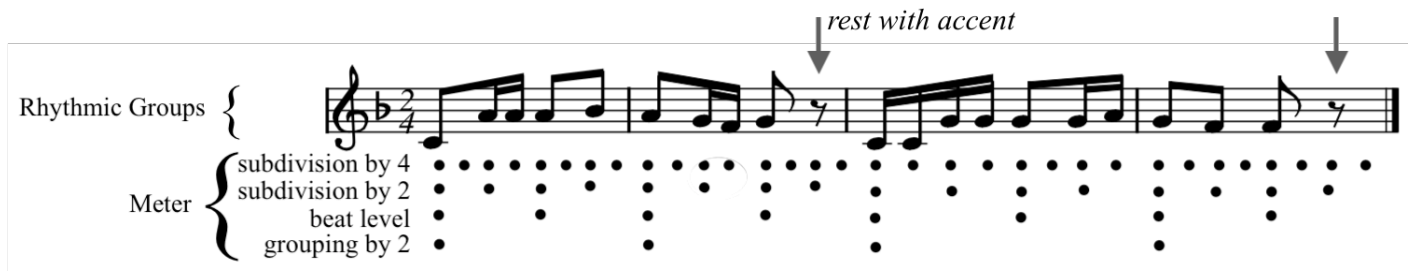

Figure 1. The notes of a rhythmic group can be organized into a metrical hierarchy. Each row below the musical staff represents a unique metric level, and points in time where many metrical levels align are points of metrical accent. The third row represents the beat level or the level at which most people would to the music. Events on the third row are perceived as more accented than events on the second row, which are in turn perceived as more accented than events on the first row. Metrical levels are related to one another by simple integer ratios. Figure adapted from Large and Kolen (1994). Reprinted with permission.

Oftentimes in music, the beat is implied rather than physically realized. For example, the melody in Figure 1 does not have a note at all points of metrical 
accent (i.e., the rest at the end of each bar), and yet a beat is still perceived there (i.e., the continuation of the "subdivision by 2" metrical level). As such, meter is best understood as serving a predictive function, guiding the listener in developing expectancies about upcoming events. The sensation of rhythm arises from the interaction between rhythmic groups and the meter (London, 2012). Rhythm is a central aspect of the perception and production of music.

A rhythmic group can have many or few notes that align with the meter, creating different amounts of congruence (or tension) between them, giving a rhythm its characteristic feel. Syncopated rhythms are rhythms that are unexpected in some manner. They tend not to have notes at points of metrical accent. As such, a syncopated rhythm disrupts the expectancies that are created by the underlying meter, making it more unpredictable. Moderate levels of this kind of rhythmic complexity ${ }^{2}$ have been found to increase feelings of groove (Sioros et al., 2014; Witek et al., 2014) without compromising movement synchronization (Witek et al., 2017), as compared with low levels of syncopation.

\section{Acoustic Properties of Groove}

Various acoustic features of musical stimuli have been previously associated with groove. Madison et al. (2011) found that beat salience and event variability ${ }^{3}$ were both associated with groove ratings. Faster tempos have also been associated with groove (Janata et al., 2012). By analyzing the corpus of musical examples used in Janata et al. (2012), Stupacher et al. (2016) identified event variability, spectral flux, and $\mathrm{RMS}^{4}$ variability as features that were predictive of groove ratings. Additionally, they found that songs with a drum kit differed significantly from songs without a drum kit on all features studied, suggesting

\footnotetext{
${ }^{2}$ Another measure of rhythmic complexity, joint audio entropy, has not been found to be related to ratings of wanting to move (Witek et al., 2014).

${ }^{3}$ Event variability is called "event density" in Madison et al.'s (2011) original article. See the introduction of Study 3 for more details.

${ }^{4} \mathrm{RMS}$ (root mean square) is a measure of the total energy present in a waveform. It is calculated by (a) squaring each value in the waveform, (b) averaging the values at all time points, and (c) taking the square root of the result.
} 
a role of instrumentation.

Stupacher et al. (2016) also investigated the effects of frequency range on the ability to accurately and consistently tap along with music. They experimentally manipulated the frequency of the bass drum and the bass and asked participants to tap along to the beat as synchronously as possible. They found that tapping was less variable and more forceful when the bass instruments had lower frequencies. Groove ratings were also higher for these excerpts, suggesting a role of low-frequency energy.

Burger et al. (2012) used motion capture to elucidate the relationship between acoustic features of music and spontaneous full-body movement. Participants listened to 30-second excerpts of music and were asked to move in a way that felt natural, and were encouraged to dance if they wanted. Correlation analyses revealed that music with a clear pulse and with high percussiveness elicited movements with low spatial variation ${ }^{5}$, indicating that participants' movements were simpler and exhibited fewer degrees of freedom. Temporal regularitymovement at the same frequencies as the meter - was related to these features as well as low-frequency spectral flux. In a second study, they found that pulse clarity was related to regularity in movements of all body parts, whereas spectral flux and percussiveness were more related to specific body parts such as the head and hands (Burger, Thompson, Luck, Saarikallio, \& Toiviainen, 2013). If these acoustic features are characteristic of music that supports synchronous movement, it seems likely that they would also be characteristic of music that compels synchronous movement, as in groove.

\footnotetext{
${ }^{5}$ Spatial variation is a measure of the intrinsic dimensionality of a data set. More variables are required to represent more complex data sets, and would thus be considered to have higher spatial variation. For example, given a principle components analysis of some data, a measure of spatial variation could be the number of components required to explain a certain amount of variance.
} 


\section{Neural Entrainment to Music}

Music is able to entrain the firing patterns of neurons, and many studies have found that neurons entrain their firing to both isochronous (Arnal, Doelling, \& Poeppel, 2015; Fujioka et al., 2012; Nozaradan, Peretz, Missal, \& Mouraux, 2011; Snyder \& Large, 2005) and non-isochronous (Nozaradan, Peretz, \& Mouraux, 2012; Nozaradan, Zerouali, Peretz, \& Mouraux, 2015) rhythms. These studies used various methods including electro- and magnetoencephalography (EEG and MEG), transcranial magnetic stimulation (TMS), and functional magnetic resonance imaging (fMRI). Features of the E/MEG that have been investigated include the steady-state evoked potential (SSEP), spectral activity in the beta and gamma frequency ranges, and inter-trial phase coherence. These methods and results are presented in the following sections.

\section{Steady-State Evoked Potential (SSEP)}

Nozaradan et al. $(2012,2011,2015)$ published a series of articles using a novel method for studying spectral activity at beat-related frequencies in the EEG, called the SSEP (see Nozaradan, 2014 for a review). To calculate the SSEP, multiple trials are averaged together in the time domain in order to discard non-phase-locked activity. A fast-Fourier transformation (FFT) is performed on each channel ${ }^{6}$ of EEG data and the spectra of all channels are averaged together. The resulting averaged spectrum is then inspected at frequencies of interest to determine the extent of entrainment.

In a first study, participants listened to a pure tone that had been amplitude modulated to have a main pulse of $2.4 \mathrm{~Hz}$ (Nozaradan et al., 2011). In the EEG there was a significant peak in the SSEP at $2.4 \mathrm{~Hz}$ (Figure 2, top panel), suggesting that neural activity was being entrained by the stimulus. Participants were also instructed to imagine duple or triple meter (i.e., by perceptually grouping the beats in twos or threes). This resulted in additional peaks at frequencies

\footnotetext{
${ }^{6} \mathrm{~A}$ channel here refers to the time-series data from a single EEG electrode.
} 
associated with these meters (Figure 2, middle and lower panels), suggesting that the SSEP can capture entrainment that is endogenously generated. That is, energy in frequencies associated with the imagined meter were not present in the stimulus, and must have been generated by the neurons themselves. When participants biased their perception of the stimulus, this was reflected in the EEG.
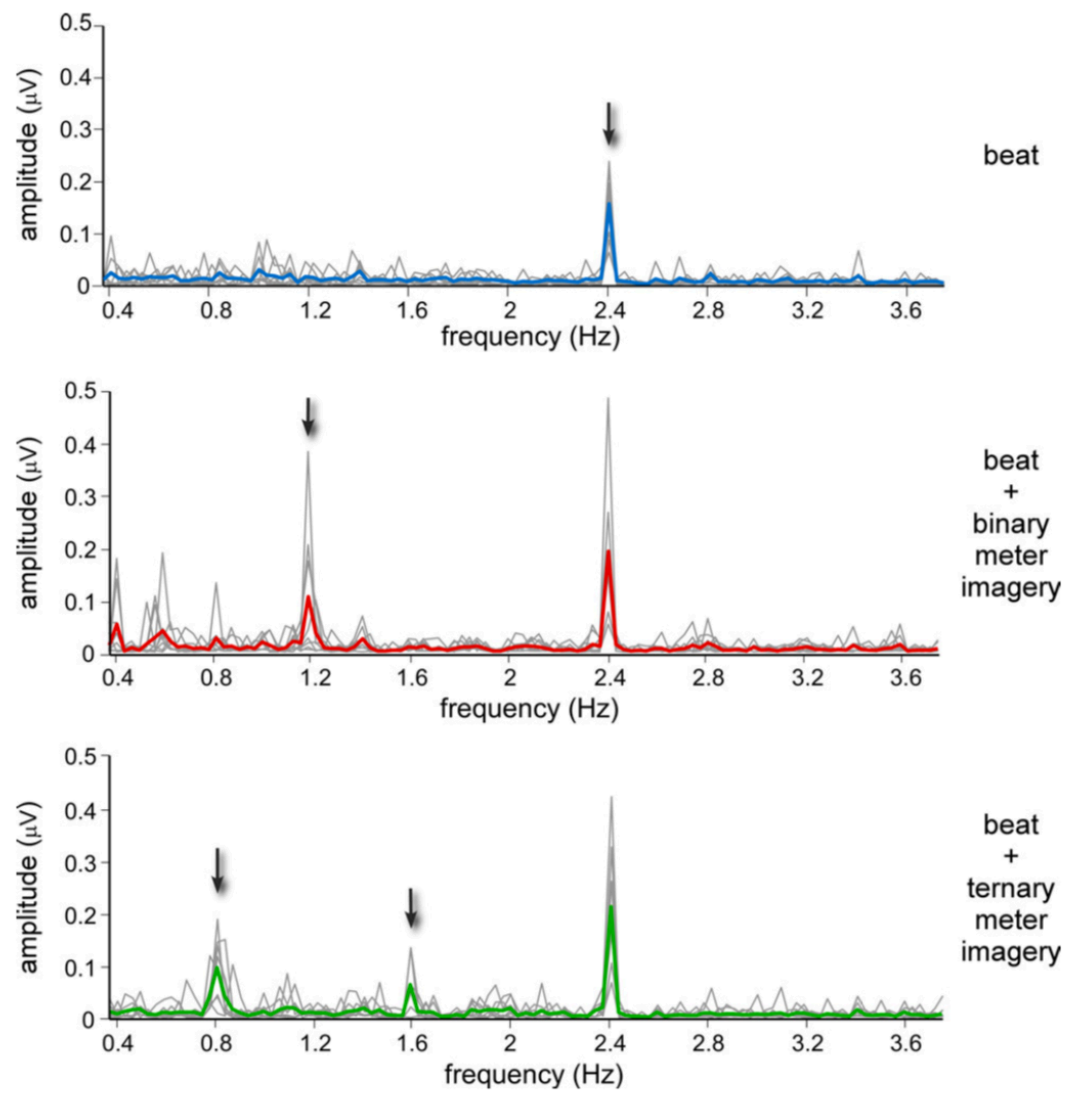

Figure 2. Steady-state evoked potentials. When participants listen to a periodic stimulus with a beat rate of $2.4 \mathrm{~Hz}$, there is a peak in the SSEP at $2.4 \mathrm{~Hz}$ (top panel). When they imagine grouping beats in twos $(1.2 \mathrm{~Hz}$; middle panel) or threes $(0.8$ and $1.6 \mathrm{~Hz}$; bottom panel), corresponding frequencies are present in the SSEP. Figure from Nozaradan et al. (2011). Reprinted with permission.

The SSEP is also sensitive to meter. When participants listened to rhythms which contained more than one periodicity, the SSEP reflected this with peaks 
at beat- and meter-related frequencies (Nozaradan et al., 2012). It is important to note that the frequencies being considered here are not necessarily those that are most prominent in the physical stimulus, but rather more "musical" ones that would be used for finding the beat and tapping your foot. Interestingly, SSEP activity at these frequencies was selectively enhanced, irrespective of the spectrum of the stimulus. Thus, there does not seem to be a one-to-one mapping between the spectrum of the stimulus and the SSEP, indicating that a sense of beat and meter are (at least partly) endogenously generated.

The SSEP has also been found to track the beat and meter in natural music, and this tracking is disrupted in the presence of contradictory rhythmic cues. Tierney and Kraus (2014) played a pop song for participants with a superimposed metronome that was either aligned with the beat or shifted away by $25 \%$. Critically, there were only negligible differences in the spectra of the stimulus for the on-beat and off-beat conditions. Neural entrainment to the beat frequency was much reduced when the metronome was not aligned with the beat, despite the fact that there was the same amount of energy in the stimulus at that frequency. This suggests that neurons are not only entraining to the stimulus, but are also sensitive to the metrical structure of the input.

\section{Frequency Bands}

While the SSEP only concerns relatively low frequencies (e.g., $<4 \mathrm{~Hz}$ ), higher frequency bands have also received some attention with regards to neural entrainment. M/EEG activity has long been categorized into different frequency bands, defined loosely as follows: delta $(<4 \mathrm{~Hz})$, theta $(4-7 \mathrm{~Hz})$, alpha (8-12 $\mathrm{Hz})$, beta $(13-30 \mathrm{~Hz})$, and gamma $(>30 \mathrm{~Hz})$. In auditory cortex, studies suggest that these bands are hierarchically organized, whereby neural oscillations in lower frequency bands (e.g., delta) can modulate the amplitude and excitability of higher frequency bands (e.g., gamma; Lakatos et al., 2005). Thus, synchronized neural firing at gamma frequencies becomes more likely at certain points in time, due to the underlying delta oscillation. This system has been suggested 
to underlie attentional selection, where rhythmic sensory streams can entrain delta oscillations and modulate task performance (Lakatos, Karmos, Mehta, Ulbert, \& Schroeder, 2008).

To assess entrainment of different frequency bands, a time-frequency spectrum (usually a wavelet analysis) is used to assess spectral changes over time. To assess entrainment, the spectrum can be averaged across a specific range of frequencies (such as 13-25 Hz, as in Figure 3 panel F), and the spectrum of the result can be assessed at the beat frequency of interest. In beat perception research, the frequency bands that have received the most attention are gamma (Snyder \& Large, 2005) and beta (Fujioka et al., 2012).

Using EEG, gamma-band activity calculated over fronto-central electrodes has been found to synchronize with an isochronous sequence of loud and soft tones (Snyder \& Large, 2005). Additionally, phase-locked (evoked) gamma-band peaks arrived late when a tone was omitted, whereas non-phase-locked (induced) peaks remained synchronous with the meter (Zanto, Snyder, \& Large, 2006). This suggests that induced gamma-band activity indicates the prediction of the temporal location of upcoming beats.

Using MEG, the modulation of beta-band activity has been found to synchronize with isochronous sequences at various rates (Fujioka et al., 2012). Specifically, there is a consistent decrease in beta-band power after each tone (Figure 3), reaching a minimum at $200 \mathrm{~ms}$ post-stimulus onset. This decrease is followed by an increase in power that peaks concurrently with the onset of the next tone, regardless of the repetition rate. Beta-band activity is possibly of interest to the current dissertation due to its association with movement (Engel \& Fries, 2010).

\section{TMS and Motor Cortex Excitability}

Transcranial magnetic stimulation (TMS) has been used to investigate neural response to rhythm. Using TMS, the primary motor cortex can be rhythmi- 

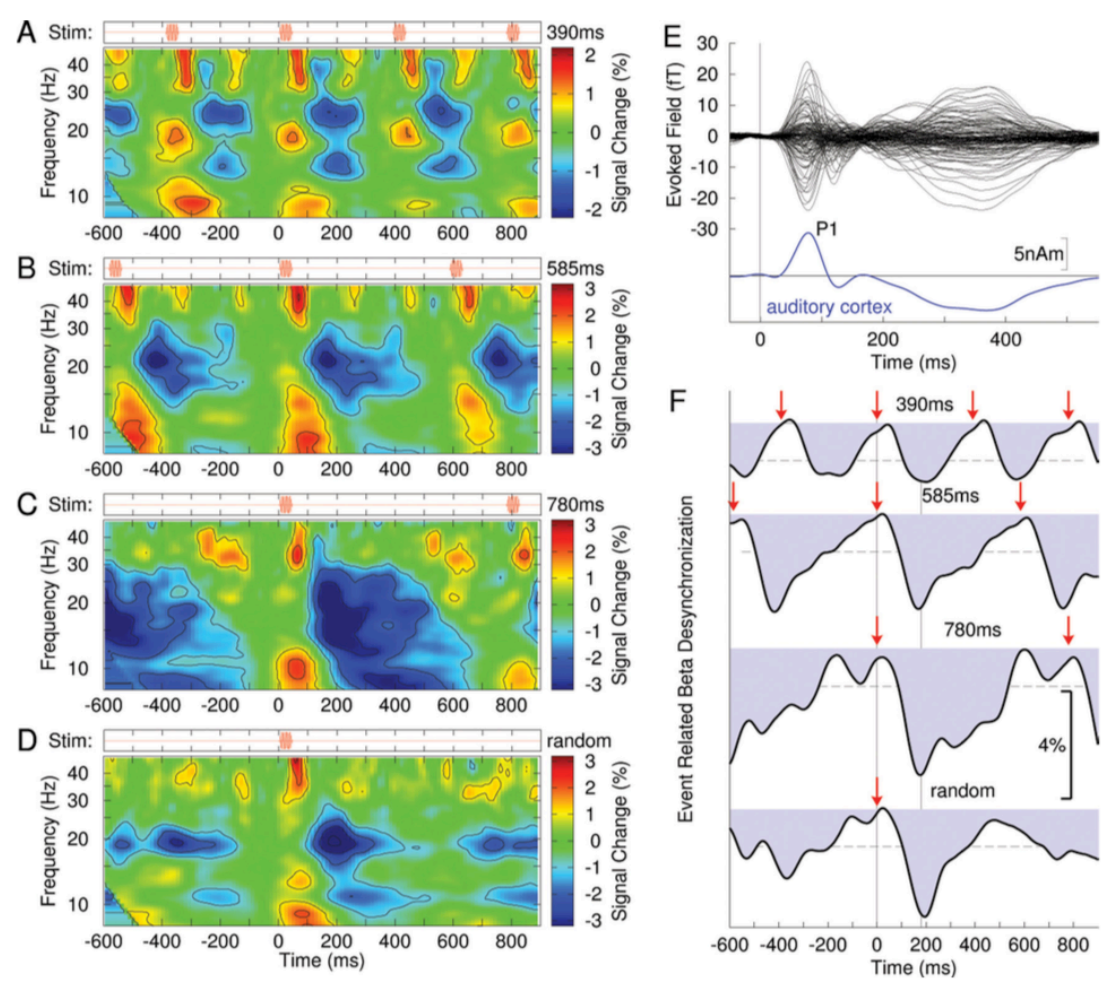

Figure 3. Beta-band entrainment to a metronome. Spectrogram of MEG activity while listening to an isochronous sequence at different rates (panels A-D). Beta-band activity $(13-25 \mathrm{~Hz}$ ) decreases $200 \mathrm{~ms}$ after each note onset (panel F). The increase in activity that follows is timed to the onset of the next beat. Red arrows indicate note onsets. Figure from Fujioka et al. (2012). Reprinted with permission. 
cally stimulated with an isochronous pulse while the participant is listening to music. Electromyography (EMG) can then be used to measure peripheral muscle activation corresponding to the region of stimulation in the motor cortex. Stupacher et al. (2013) found that when the TMS pulses are presented on the beat of the music, the resulting motor evoked potentials (MEPs) are greater than when the pulses are presented off the beat. This suggests that neurons may already be preparing to fire during on-beats, resulting in a large increase in MEPs. By contrast, during off-beat TMS pulses neurons are not preparing to fire, and so the increase in MEPs is not as large. This study also suggests a role of the motor system in beat perception.

\section{Localization}

What parts of the brain are involved in neural entrainment to the beat of music or other auditory stimuli? Auditory regions have been implicated, but also many other regions including PMC, basal ganglia (BG), and visual regions (Escoffier, Herrmann, \& Schirmer, 2015; Fujioka et al., 2012; Grahn \& Brett, 2007; Grahn $\&$ Rowe, 2009). It is surprising in some respects that non-auditory regions would be attuned to an auditory stimulus. One potential explanation is that if one sensory modality detects some regularity in the world, other brain regions would be interested in this so that they can interact with the world appropriately. For example, motor cortex would need to know the temporal location of beats in order to synchronize movements with it.

Grahn and Brett (2007) used fMRI to study the brain regions involved in the perception of a beat in musical rhythm. They also measured participants' ability to tap along with different rhythms, and found that performance was better for rhythms that had a stronger sense of meter. These same rhythms elicited a greater BOLD ${ }^{7}$ response in the BG and SMA. Grahn and Rowe (2009) found that the presence of a clear beat was associated with greater connectivity be-

\footnotetext{
${ }^{7}$ The blood oxygenation level dependant (BOLD) response is a measure of blood flow that is measured via functional magnetic resonance imaging (fMRI).
} 
tween BG, PMC, and auditory cortex. While these studies were not able to investigate entrainment of neural populations per se, it seems that beat perception recruits areas of the brain related to movement.

While fMRI work dominates the literature with regards to localization due to its excellent spatial resolution, addressing the question of localized entrainment requires a brain imaging technique that also has good temporal resolution. MEG and spatial filtering (i.e., with a beamforming algorithm) have been used for this purpose (Fujioka, Zendel, \& Ross, 2010). For example, Fujioka et al. (2012) used MEG to search for brain regions with beta-band oscillations that were phase coherent with auditory cortex, and found the strongest coherence in the cingulate gyrus, close to SMA.

With EEG, source analysis is becoming increasingly feasible with the advent of high-density systems (i.e., 64+ channel recordings). Independent components analysis (ICA) with dipole fitting can be used to localize activity measured via EEG (Makeig, Debener, Onton, \& Delorme, 2004; Onton, Westerfield, Townsend, \& Makeig, 2006). ICA is a method of identifying the activity of many sources whose activity has been linearly mixed onto a number of sensors (Bell \& Sejnowski, 1995). In the case of EEG, there are many sources of electrical activity in the brain, whose activity is measured at all scalp electrodes in different combination (Bell \& Sejnowski, 1995; Makeig, Bell, Jung, \& Sejnowski, 1996). ICA decomposes the sensor data into a number of possible independent components (ICs) that model the time-series activity of possible independent sources. To localize the ICs, a dipole model can be fit to its scalp topography. Since brain sources are dipolar (Delorme, Palmer, Onton, Oostenveld, \& Makeig, 2012), ICs with well-fitting dipole models (i.e., dipole models with low residual variance) are likely to originate from a single source in the brain. The location of the center of the dipole model is considered to be the location of the source of activity.

The validity of ICA rests on a few reasonable assumptions with respect to EEG, namely that the sources are independent from one another, and that transmis- 
sion delays from the source to the sensor are negligible. Additionally the number of ICs returned cannot exceed the number of sensors, but when the number of sensors is high it has been shown that ICA can separate out the contributions of dozens of maximally independent information sources (Makeig et al. (2004), 71 sensors). The accuracy of this method has been corroborated in EEG studies replicating sources found in functional magnetic resonance imaging (fMRI) studies (McGarry, Russo, Schalles, \& Pineda, 2012), as well as simultaneous fMRI-EEG studies (Yin, Liu, \& Ding, 2016).

\section{Neural Mechanisms}

Neural resonance theory (Large \& Snyder, 2009; Large, Herrera, \& Velasco, 2015) offers a potential mechanistic account of how neurons synchronize with the beat. The theory posits that neurons act as frequency-tuned oscillators that will automatically synchronize their firing with the incoming sensory stream. Different neurons will entrain to different metrical levels, creating a neural representation of the different patterns of oscillatory activity in the stimulus. Critical for the theory is that neurons are connected in multiple hierarchical levels, where the firing of higher levels will automatically entrain (or resonate) to the firing patterns in lower levels. At points of metrical accent, many neurons at lower levels will be firing at the same time, and will give rise to stronger synchronization at higher levels. In this way, a sense of the beat and the accompanying meter can emerge as a natural consequence of the periodicities present in the stimulus.

This representation of meter in higher-level neural populations can be considered a "model" of the stimulus, different from the more "verbatim" representation that is likely present in primary sensory cortices. As such, the brain can use this model to make predictions about the stimulus, for example the temporal location of future beats. This conceptualization bears some resemblance to predictive coding theory (Friston, 2010), which states that the brain creates internal models of sensory input based on experience. By continually comparing the pre- 
diction with actual sensory input, the brain is able to evaluate its performance and update the model accordingly. Thus, all sensory input is perceived in the context of models which already exist based on one's previous experiences with similar stimuli. In the case of beat perception, rhythms will be perceived based on the statistical regularities present in music (Vuust \& Witek, 2014). For example, since most Western music is in duple meter (where beats are grouped in twos), an isochronous sequence without any physical metrical accents will often be perceived as having a duple meter (Smith, Cuddy, \& Upitis, 1994). It has been proposed that duple meter may arise in many cultures as a function of established internal models of locomotion which are constrained by bipedalism (see Mithen (2005), pp. 150-154).

Because of the "automatic" nature of neural resonance, it seems likely that any two networks in the brain could spontaneously fire together provided that a) they are physically connected in some way and b) the preferred patterns of firing are similar. Taken together with predictive coding, this suggests neural models developed for one input source could resonate with input from another source. PMC for instance, which is well-connected with auditory cortex (Zatorre, Chen, \& Penhune, 2007) and likely shaped by experience with locomotion, may have a direct role in predicting auditory sensory input (Merchant, Grahn, Trainor, Rohrmeier, \& Fitch, 2015; Patel \& Iversen, 2014). Put into a neural resonance framework, existing oscillatory motor programs (e.g., for walking) that match the auditory sensory input will increase the amplitude of their oscillation, which

will feed back to auditory regions to create a temporal expectancy for an upcoming auditory events. What is interesting about this case is that a motor program that ostensibly developed to support locomotion falls into the service of auditory perception.

\section{The Groove Pathway}

Taken together, it seems possible to chart the pathway through the brain from the sensation of music to the feeling of groove. Music enters the ear and is 
analyzed by the auditory system by synchronizing neural firing patterns with the periodicities present in the rhythm and meter. These entrained oscillations are the brain's preliminary model of what it is sensing. Because this firing is periodic, the oscillations begin to be generated endogenously and are thus able to make predictions about how the music will continue to unfold. Based on the rhythm and the implied meter, many nested oscillations at different frequencies will exist, creating a unique periodic signature for this particular music. Other systems in the brain that are accustomed to this pattern of oscillations will begin to resonate with the signal as well, thus lending their expertise for modeling the input and making predictions.

As previously mentioned, the idea that the motor system would be called upon to aid with auditory prediction is not new (Patel \& Iversen, 2014), and it seems particularly well-suited to music. Humans are bipedal, so the motor plan for walking is based on groupings of two, which matches with meters that are commonly found in music systems around the world (e.g., 2/4 or 4/4). Even when the meter has groupings of three (e.g., triple meter, 3/4,6/8), the underlying subdivision is typically in two. Compound meters (e.g., 9/8), where the subdivision is also three, are exceedingly rare. The prevalence of the grouping by twos in music may all relate back to the body and bipedalism. Thus, for the listener, it would make sense to use this same motor system to aid with the perception of music when listening.

When the motor system resonates with the input because there is an existing motor program that is a good match, the brain is essentially running this program as if it were preparing to move. This motor plan becomes part of the internal model of the auditory input; the brain's ability to move the body is guiding and constraining its predictions about music. Different pieces of music can engage the motor system in different ways and to different degrees. And only when motor plans are sufficiently engaged could the body begin to feel a pull towards fulfilling that plan and actually moving in accordance with it. 


\section{Research Aims}

The current dissertation investigated the effects of psychological, musical, and acoustical features of music on neural entrainment of the motor system. Study 1 concerned the effects of groove on neural entrainment in PMC, using a subset of stimuli that have been rated on amount of perceived groove (Janata et al., 2012). Study 2 concerned the musical feature of syncopation, which is related to a sense of groove (Witek et al., 2014). Clave rhythms with varying degrees of syncopation were played for participants to investigate its effects on movement synchronization and neural entrainment. Study 3 concerned acoustical features of music that have been found to contribute to groove, and investigated their effects on movement synchronization and neural entrainment. 
Study 1: Psychological Aspects of Music (Feelings of Groove)

The ability to perceive a beat in music is a relatively ubiquitous skill, whether the rhythms are simple and on-beat or complex and syncopated. Neural oscillations are thought to underlie this ability (M. J. Velasco \& Large, 2011), and studies using M/EEG have demonstrated neural entrainment to the beat frequency of isochronous (Fujioka et al., 2012; Snyder \& Large, 2005) as well as complex (Nozaradan, Peretz, \& Keller, 2016; Nozaradan et al., 2015) rhythms. Similar evidence has also been found in infants, underscoring the ubiquity of the propensity to develop the ability to perceive a beat in music (Cirelli, Spinelli, Nozaradan, \& Trainor, 2016; Honing, Ladinig, Háden, \& Winkler, 2009). One proposed mechanism for neural entrainment to the beat is neural resonance (Large \& Snyder, 2009), which posits that neurons synchronize their firing with periodicities present in the stimulus. In this way, the brain is able to generate a model of the stimulus and make predictions about how the stimulus will unfold over time. This neural entrainment also appears to be cross-modal, such that auditory oscillations may entrain visual cortices, for example (Escoffier et al., 2015). Thus, neural entrainment may be thought of as a global mechanism by which the brain is able to make useful predictions about timing of future events.

Theories of predictive coding suggest that the brain creates a model of sensory input, and that this model is used to generate predictions about future events (Friston, 2010). The brain will work to match the incoming sensory information with some existing model by maximizing the mutual information between them. By continually comparing sensory input with the output of the model, the brain is able to evaluate its performance, and any prediction errors may be used to update the model (Vuust \& Witek, 2014). In beat perception, motor planning areas are thought to be involved in predicting the timing of future beats (Chen et al., 2008; Patel \& Iversen, 2014). Patel and Iversen (2014) propose that motor planning regions draw on their experience with periodic movement (e.g., walking) by simulating these movements in an effort to predict future auditory events. This would suggest that neural models of music and movement are 
similar, at least for music where a beat can be perceived.

Given the oscillatory nature of neurons and musical beats, the neural resonance framework provides a plausible mechanism for communication between sensory (i.e., auditory) and motor areas of the brain. In particular, the oscillatory activity of a motor program that corresponds with the oscillatory activity in auditory areas will increase via synchronization of neuronal populations. The output from this motor program may then feed back to auditory areas to create a temporal expectancy for a future auditory event.

Brain imaging work has found support for the idea that the motor system is involved in beat perception. Using MEG, Fujioka et al. (2012) found that while listening to isochronous sequences, power in the beta-band oscillated in a manner that was synchronized with the stimulus. Using a beamforming localization procedure, they found significant beta-band phase coherence between primary auditory areas and supplementary motor area (SMA). Using fMRI, Grahn and Brett (2007) found that rhythms that are easier to reproduce by tapping also elicited more activity in motor planning areas such as SMA and BG.

If motor planning areas are activating certain motor programs while listening to rhythms, it is also possible that this activation will prime movement that is associated with that motor program. "Groove" is a high-level musical feature referring to music that compels synchronous movement (Janata et al., 2012; Madison, 2006; Witek et al., 2014). Consistent with this idea, Janata et al. (2012) found that participants' tapping was temporally aligned with the periodicities present in a musical excerpt when the excerpt was considered high in groove (as rated by a separate group of participants). This suggests that the neural resonance of motor planning areas with auditory areas is greater when it is driven by music that is high in groove.

Stupacher et al. (2013) found evidence in support of the notion that groove preferentially engages the motor system. Participants listened to high- or low-groove music while single-pulse TMS was applied over primary motor cortex either onbeat or off-beat. This procedure elicits motor-evoked potentials (MEPs) in the 
corresponding muscles of the body, in this case the arm. Surface electrodes on the arm were used to measure the magnitude of MEPs in response to the TMS pulses. They found that MEPs were greater while participants listened to high-groove music, suggesting that the motor system was preferentially engaged. Thus, the potential effects of high-groove music may encompass primary motor cortex as well as motor planning areas, preparing the body to move in a manner that is temporally aligned with the stimulus.

The current study sought to extend the findings of Stupacher et al. (2013) by investigating whether entrainment to the beat frequency in motor planning areas would differ for high- versus low-groove music. Musical excerpts varying in amount of perceived groove (Janata et al., 2012) were presented to participants while EEG data were recorded. EEG data were subjected to ICA to identify ICs of activity. Next, dipole models were used to select ICs that were within regions of interest (premotor, auditory, and visual cortices). Neural entrainment to the beat frequency was measured for all ICs. It was predicted that premotor ICs would entrain to the beat frequency of all stimuli, but show the greatest entrainment for high-groove music and the lowest for low-groove music. Although we did expect auditory and visual ICs to entrain to all stimuli, we did not predict a difference in entrainment for music with different amounts of groove.

\section{Methods}

\section{Participants}

Eleven participants with self-reported normal hearing were recruited from the Ryerson University psychology student participant pool. Based on self-report, two were left-handed, three had perfect pitch, seven considered themselves musical, and six were musically active at the time of testing (playing or singing in a group or on their own). They had an average of 7 years of musical experience with a range of 1 to 18 years. This study was approved by the Ryerson Research Ethics Board and all participants completed informed consent prior 
to the study.

Based on the achieved effect size of previous work $\left(\eta^{2}=0.62\right.$; Nozaradan et al., 2011), a sample size calculation revealed that a $n$ of four would be necessary.

\section{Stimuli}

Thirty pieces of music representing high- medium- and low-groove were selected on the basis of ratings provided by Janata et al. (2012) Excerpts were restricted to the first 30 seconds of each piece on the basis of previews available on the iTunes Music Store (Apple, Inc.). The tempo of each excerpt was extracted manually by the first author. Seven excerpts had tempi that changed over the course of the excerpt and were thus excluded from analysis. ${ }^{8}$ See Table 8 in Appendix A for a complete list of the excerpts used.

\section{Procedure}

Participants were seated at a computer in a sound-attenuated chamber. Stimulus presentation was controlled using Presentation software (Neurobehavioral Systems) running on a PC. Excerpts were played over Logitech Z130 loudspeakers in two blocks. Each block contained one presentation of each excerpt. The order of presentation within blocks was individually randomized. Participants pressed the "space" bar on the keyboard to initiate each excerpt. Participants were asked to listen to each excerpt carefully but to refrain from moving.

EEG data were recorded using a 64-channel BioSemi ActiveTwo system and were digitized at $512 \mathrm{~Hz}$. Analyses were carried out using a combination of custom MATLAB (version R2016a; The Mathworks, Inc.) scripts and EEGLAB (version 13.5.4b; Delorme \& Makeig, 2004).

Preprocessing and Independent Components Analysis. To improve the quality

\footnotetext{
${ }^{8}$ This was determined (unfortunately) after data had already been collected. Corrections for the possibility of heterogeneity of variance are presented below.
} 
of the ICA decomposition, various preprocessing steps were executed (I. Winkler, Debener, Muller, \& Tangermann, 2015). Low-frequency drift is a common non-stationary artifact, which has the potential to detract from the reliability and dipolarity of the ICs (Debener, Thorne, Schneider, \& Viola, 2010). Lowfrequency drift can be removed with a high-pass filter at $1 \mathrm{~Hz}$ or above (I. Winkler et al., 2015). However, since we were interested in beat frequencies near $1 \mathrm{~Hz}$, two preprocessing pipelines were necessary: one with a high-pass filter at $1 \mathrm{~Hz}$ to obtain the ICA weights, and a second with a high-pass filter at $0.1 \mathrm{~Hz}$ to obtain activity within each electrode. The ICA weights from the first pipeline were then used with the preprocessed electrode activity from the second pipeline in order to obtain IC activity that was free of low-frequency drift.

For the first pipeline, raw EEG data were high-pass filtered at $1 \mathrm{~Hz}$ using the EEGLAB function pop_eegfiltnew. Bad channels were identified using the clean_rawdata plugin. This plugin works by first windowing the data into 1second non-overlapping windows. In each window, each channel is interpolated based on all other channels, and this interpolation is correlated with the actual data from that channel. If this correlation is lower than 0.8 , the channel is flagged for the given window. Any channel with flags in more than half of the windows is rejected. Data were then rereferenced to the average, and line noise was removed (pop_cleanline). Sections of data where no music was presented (i.e., while entering responses on the computer) were removed (pop_epoch), and the remaining data were subjected to the extended version of the Infomax ICA (Bell \& Sejnowski, 1995) as implemented in EEGLAB (pop_runica).

In the second pipeline, raw EEG data were high-pass filtered at $0.1 \mathrm{~Hz}$. Channels that were identified as bad in the first pipeline were removed. All other channels were referenced to the average and epoched. Next, the ICA weights and sphering matrices (i.e., EEG.icaweights and EEG.icasphere) obtained from the first pipeline were used with the electrode data from the second pipeline to compute IC activity. 
Regions of interest were defined based on Brodmann areas (BA): the PMC region comprised BA6, the auditory cortex region comprised BA41, BA42, and BA22, and the visual cortex region comprised BA17, BA18, and BA19. In order to localize components with respect to these target brain regions, equivalent dipole models were fit to each IC (pop_dipfit). ICs were not considered for analysis if the residual variance of its corresponding dipole model was less than $15 \%$. For each region of interest, an IC was selected if its Talairach coordinates were within $8.66 \mathrm{~mm}^{9}$ of the target brain region, using the Talairach Daemon (Lancaster et al., 1997, 2000).

Entrainment Analysis. The following steps were implemented to determine extent of entrainment in each IC:

1. The activity in the two presentations of each excerpt was averaged in the time domain (to reveal phase-locked activity) and subjected to a fast Fourier transform with bin width of $0.0312 \mathrm{~Hz}$.

2. The noise floor was removed from each bin by subtracting activity in the surrounding bins as in Nozaradan et al. (2011).

3. The extent of entrainment was defined as the maximum value across the eleven frequency bins centered on the beat frequency ${ }^{10}$. A $t$-test was used to ensure that this value was significantly greater than zero (i.e., that the peak was above the noise floor).

For participants with multiple sources localized in the same region, only the maximum entrainment value was subjected to analysis. For participants without any sources localized to a given region, the entrainment value was set to zero (i.e., no entrainment in that region).

\footnotetext{
${ }^{9}$ The localization procedure creates a 3D cube centered on the given Talairach coordinates. The cube extends $5 \mathrm{~mm}$ on all sides resulting in a total size of $11 \mathrm{x} 11 \mathrm{x} 11 \mathrm{~mm}$. The furthest distance from the center of the cube to the edge (i.e., the corner of the cube) is $8.66 \mathrm{~mm}$.

${ }^{10}$ The maximum was used instead of the mean because a) the stimuli were not computationally generated, so we cannot assume the tempo was perfectly consistent throughout each excerpt, and b) the tempo was extracted manually for each excerpt and so we cannot assume they are exact.
} 


\section{Results}

For the PMC region there were 2 participants for whom there was no IC found, and for the auditory cortex region there were 5 participants. These cells were assigned a value of zero. All remaining entrainment scores were significantly above the noise floor (See Table 9 in Appendix A).

A one-way ANOVA with Brain Region (Premotor, Auditory, and Visual) as the within-subjects factor revealed that the magnitude of entrainment differed across brain regions $(F(2,20)=15.73, p<.001)^{11}$. Visual had the highest level of entrainment $($ mean $=0.0266 \mu \mathrm{V})$ followed by Premotor $($ mean $=0.0168 \mu \mathrm{V})$ and Auditory $($ mean $=0.0075 \mu \mathrm{V})$. Because of the large differences in magnitude of entrainment across brain regions the effect of groove category was assessed using three one-way ANOVAs.

Groove Category had a significant effect in the Premotor Region $(F(2,20)=$ $5.156, p=.015$ ), wherein entrainment was greater for high-groove music (mean $=0.0176 \mu \mathrm{V})$ and mid-groove music $($ mean $=0.0182 \mu \mathrm{V})$ than low-groove music $($ mean $=0.0145 \mu \mathrm{V}$; Figure $4 \mathrm{a}$ ). Groove Category was not significant for Auditory $(F(2,20)=1.533, p=.24$; Figure $4 \mathrm{~b})$ or Visual Regions $(F(2,20)=$ $2.325, p=.12$; Figure $4 \mathrm{c}) .^{12}$

To explore what features of music might contribute to entrainment, we ran a series of correlations between the extent of entrainment and various acoustic features previously found to modulate groove, specifically event density, beat salience, tempo, and low-frequency spectral flux (Janata et al., 2012; Madison et al., 2011; Stupacher et al., 2016). The only significant correlations involved tempo. As illustrated in Figure 5, slower beat rates were associated with greater

\footnotetext{
${ }^{11}$ Mauchly's test indicated that the assumption of sphericity had been violated $\left(\chi^{2}(2)=\right.$ $0.474, \mathrm{p}=.035)$, but Greenhouse-Geisser corrected $p$-values were still significant $(\epsilon=0.66$, $p<.001$ ). Additionally, an ANOVA with the zero entrainment values removed (i.e., treating them as missing values) was also still significant $(F(2,23)=6.618, p=.0054)$.

${ }^{12}$ Mauchly's test for each Brain Region was not significant, indicating that the assumption of sphericity had been met. Additionally, ANOVAs were run with the zero entrainment values removed (i.e., treating them as missing values), and all gave similar results as leaving them in.
} 

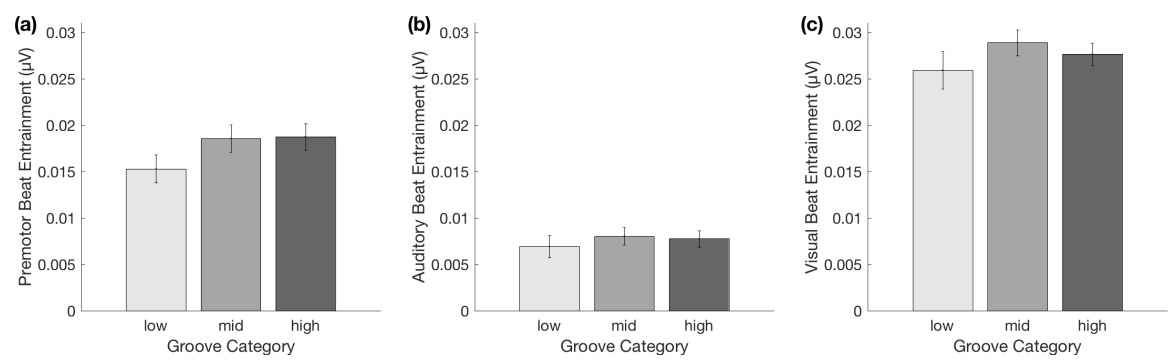

Figure 4. Neural entrainment to the beat of natural music. (a) Neural entrainment to the beat frequency in premotor cortex (BA6) was significantly greater for high- and mid-groove songs than for low-groove songs. Entrainment in (b) auditory (BA41, BA42, BA22) and (c) visual (BA17, BA18, BA19) cortices did not differ across groove categories. Error bars represent standard error.

levels of entrainment in PMC $(r=-.75, p<.001)$, Auditory $(r=-.78, p<.001)$, and Visual areas $(r=-.80, p<.001) .{ }^{13}$

\section{Discussion}

The current study found evidence for increased neural entrainment to the beat while listening to high- versus low-groove music. Importantly, this difference was present in PMC and not in auditory or visual cortices, suggesting that groove preferentially engages the motor system. This offers a potential neural basis for previous behavioural studies which have demonstrated that high-groove music elicits more synchronous tapping than low-groove music (Janata et al., 2012). Consequently, I suggest that motor output may be better synchronized with high-groove music relative to low-groove music because neural populations in PMC are better-entrained.

Viewed through the lens of the predictive coding framework (Friston, 2010; Vuust \& Witek, 2014), increased synchronous firing of PMC during high-groove music listening suggests that motor models are being prioritized somehow (over

\footnotetext{
${ }^{13}$ The correlations were also run separately within each participant. The median correlations were -.43 for PMC (range -.27 to -.62), -.46 for Auditory (range -.21 to -.52), and -.36 for Visual (range -.16 to -.60).
} 


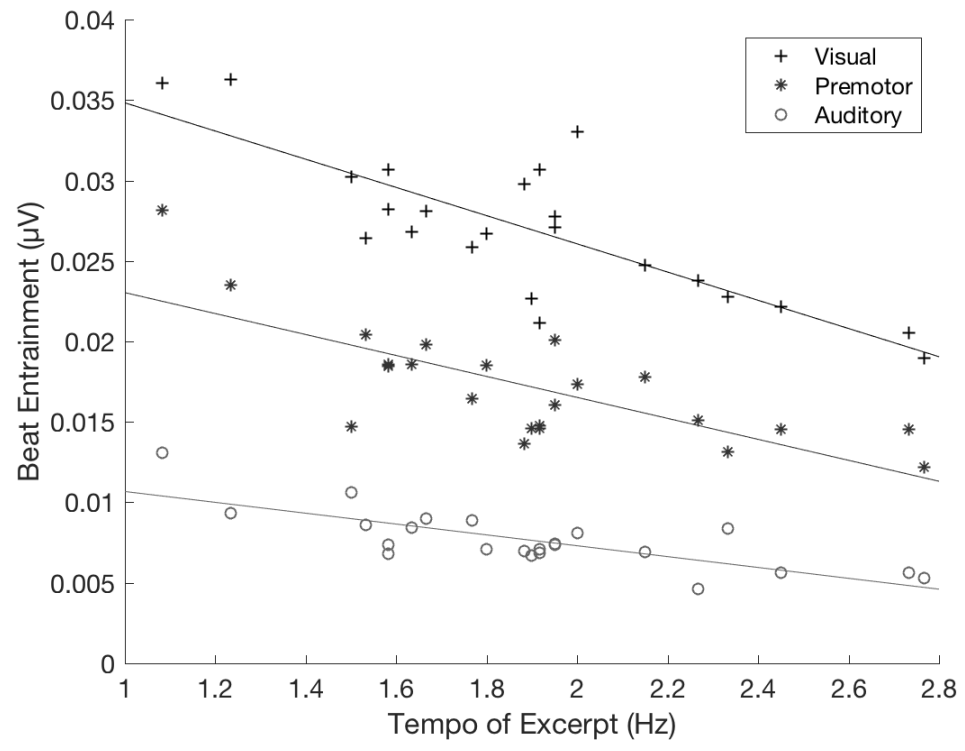

Figure 5. Correlation between neural entrainment and tempo. Neural entrainment to the beat frequency was negatively correlated with the tempo of the excerpt in all brain regions investigated. Slower beat rates were associated with greater entrainment. 
visual models, for example). The extent to which motor models become entrained may reflect the extent to which the underlying neural architecture is already wired for the periodicities in the stimulus. By extension, it seems possible that high-groove music compels movement because its rhythmic structure is similar to that of programs that control and plan movement.

The relatively strong level of neural entrainment observed in visual cortex was also somewhat unexpected. We might have expected greater levels of entrainment in auditory over visual areas given that our stimuli were auditory. We might have also expected greater levels of entrainment in PMC over visual areas because music compels movement. One possible explanation for the visual advantage may be the scale of neuronal populations involved; the visual cortex might simply have more neurons. Once entrained, the larger population of neurons may lead to a stronger response regardless of the driving modality.

It was also found that entrainment in all regions was negatively correlated with tempo, meaning that entrainment was greater for slower tempi. This is perhaps unexpected, since slower songs do not tend to be associated with groove (Janata et al., 2012). One possible explanation is that this is simply a result of $1 / f$ noise, whereby the power of EEG has been found to decrease with increasing frequency (Demanuele, James, \& Sonuga-Barke, 2007). Another explanation is that tempo has an influence on entrainment that is independent of groove, such as a preferred entrainment rate. For example, Parncutt (1994) found that when participants tapped along with rhythms at different tempi, the chosen tapping rate tended to gravitate toward 1.4-1.6 Hz. Merchant et al. (2015) found optimal neural synchronization in the putamen ${ }^{14}$ of monkeys at $1.25 \mathrm{~Hz}$. This could mean that neural entrainment is also greater at these frequencies irrespective of the level of groove. Since the tempi of the current stimuli ranged from 1.08 to $2.67 \mathrm{~Hz}$, it is possible that most of these excerpts are sufficiently faster than is optimal for neural synchronization, resulting in the negative correlation.

\footnotetext{
${ }^{14}$ The putamen is an area of the brain that has been implicated in beat perception (Chen et al., 2008; Grahn \& Brett, 2007).
} 


\section{Conclusions}

While ICA has traditionally been used for the removal of artifacts from electrode data, the current study analyzed the waveforms of the components themselves. Using dipole fitting we were able to localize these components, and were thus able to compare time-series EEG data across different regions of the brain. Results showed that PMC preferentially entrain to high-groove music. In contrast, auditory and visual cortices do not exhibit a preference for level of groove, suggesting that this is specific to motor-related areas. This study provides a neural basis for previous reports that a sense of groove is related to a desire to move along with the music (Janata et al., 2012).

However, there are many aspects of music that might contribute to a sense of groove, such as familiarity, preference, low-frequency energy (Stupacher et al., 2016), or syncopation (Sioros et al., 2014; Witek et al., 2014). Study 2 investigates the effects of syncopation in particular. In addition to assessing its influence on ratings of wanting to move, I also consider its influence on neural entrainment of PMC and tapping performance. 
Study 2: Musical Aspects of Music (Syncopation)

Music can be a compelling thing to listen to, often making us want to move along with the rhythm and synchronize our movements. Why is it that certain rhythms create this desire more strongly than others? What is it about our perception of these rhythms that is better-able to engage the motor system and increase our desire to move along? One can imagine that in order to move along with a rhythm, it must be sufficiently predictable. However, the most predictable rhythm, a metronome, seems to create less of a desire to move than would a more syncopated rhythm. If you ask musicians to play a melody in a manner that maximizes groove (the desire to move), they will make the rhythm of the melody more syncopated (Madison \& Sioros, 2014). How does syncopation compel movement?

Several studies have found that degree of syncopation and feelings of wanting to move have an inverted-U relationship (Sioros et al., 2014; Witek et al., 2014), where rhythms that are syncopated too little or too much result in a reduced desire to move. Witek et al. (2014) collected drum breaks that varied in their degree of syncopation, and found that participants' highest ratings of "wanting to move" occurred for rhythms that had a moderate degree of syncopation. Sioros et al. (2014) adjusted the note onsets of simple piano melodies to be more or less syncopated, and also found that moderate degrees of syncopation elicited the highest groove ratings from participants.

One way of interpreting this pattern of findings is with regard to predictive coding models (Vuust \& Witek, 2014). Upon obtaining sensory input (hearing a rhythm), the brain attempts to develop a model (the meter) with which to predict how the rhythm will unfold. Some models could already exist in the system, as would be the case for common rhythms in common meters such as $4 / 4$ time (grouping beats in fours). If the model is not a perfect match to the input (there is some prediction error), then the system will attempt to update the model. 
Vuust and Witek (2014) suggest that this prediction error is what makes moderately-syncopated rhythms induce the desire to move in a listener. For rhythms with low syncopation the model perfectly fits the input and so there is no prediction error. For rhythms with high syncopation the system is unable to come up with a suitable model at all, and reverts to a "learning" mode where it is attempting to create a new model. Once again, there is no prediction error, since there is no model that is being compared to the input. At moderate degrees of syncopation the system is able to come up with a model, but the presence of syncopation leads to some prediction error. They suggest that dance is an example of the body enacting and thus emphasizing the model, in an attempt to minimize prediction error. By emphasizing the model a listener is increasing neural and physical activity corresponding to the intended meter. In a sense, you might say that by dancing, listeners are "forcing" themselves to hear the rhythm with the intended meter. This is borne out quite clearly in salsa dancing, where the steps of the dance are designed to coincide with the strong beats of the meter, while the clave plays a rhythm that is syncopated against the strong beats of the meter (Fitch, 2016).

Many studies have found evidence for the involvement of motor-related brain areas in beat perception. Using fMRI, Grahn and colleagues (Grahn \& Brett, 2007; Grahn \& Rowe, 2009) have found that rhythms which have a regular meter show more activation in motor-related areas of the brain, including PMC and SMA. Similar localization results have been found using MEG, where beta-band (15-30 Hz) activity seems to be related to beat perception (Fujioka et al., 2012; Mayville et al., 2001). There are relatively few studies that have investigated the neural underpinnings of syncopation perception per se, but many studies have found evidence for neural entrainment to meter-related frequencies when listening to rhythms (Nozaradan et al., 2012, 2011; Stupacher, Wood, \& Witte, 2017).

During the perception of moderately-syncopated rhythms, predictive coding theory suggests that the brain is updating an internal model of the meter. Where might this model exist in the brain? Since moderately-syncopated rhythms are 
associated with higher ratings of groove and wanting to move, perhaps these meter models exist in motor-related areas. Indeed, imaging evidence corroborates the idea that these areas are involved in supporting the perception of rhythms that can be modeled with musical meter (e.g., Grahn \& Brett, 2007).

The current study aims to investigate the effects of syncopation on neural entrainment in motor-related areas of the brain. Participants were presented with rhythms that have a low, moderate, or high degree of syncopation. They were asked to listen-only and tap along in two separate blocks, and also to rate each rhythm on how much it made them want to move along. Based on predictive coding models (Vuust \& Witek, 2014), there are a complex set of predictions for the effects of low, moderate, and high degrees of syncopation on (a) feelings of wanting to move, (b) tapping performance, and (c) neural entrainment.

For rhythms with a low degree of syncopation, predictive coding would suggest that the brain is able to summon a model that perfectly fits the input. This means that there would be very low prediction error, which should give rise to lower ratings of wanting to move and good tapping performance. Neural entrainment should be evident at the beat frequency in auditory cortex. However, on the basis of predictive coding, we might expect relatively weak entrainment in PMC at the beat frequency. This is because rhythms with low syncopation would be highly predictable and would thus have little use for a motor code to predict upcoming beats.

For rhythms with a high degree of syncopation, predictive coding suggests that the input is too complex and as such the system would not likely have a suitable model to guide predictions. If no model is identified, there is no "prediction error", which should lead to lower ratings of wanting to move, poor tapping performance, and negligible entrainment in PMC. Although I do expect auditory cortices to still synchronize with the beat frequency, I predict that there should be entrainment at many more metrical levels than for low degrees of syncopation.

For rhythms with moderate degrees of syncopation, predictive coding suggests that the brain is able to model the input, but that it is not a perfect model, and 
so there is some error between the model and the input. This may prompt an attempt to update the model based on the input. The desire to reconcile the differences between the model and the input might create a drive to reinforce one or the other. If the model in question resides in PMC, then a reasonable course of action might be to move in accordance with the model. Thus, ratings of wanting to move should be high. Since the brain is able to model the input, tapping performance should still be good, and entrainment of the beat frequency should be relatively high in PMC. I expect that entrainment in auditory cortices should be comparable to that observed for other degrees of syncopation.

To summarize, the following predictions were made. For feelings of wanting to move, results were expected to replicate Witek et al. (2014) and show an inverted-U pattern, where moderate degrees of syncopation elicit the highest ratings. For tapping performance, it was predicted that tapping variability would be highest for high degrees of syncopation, and comparable for low and moderate degrees of syncopation. For neural entrainment, it was predicted that there would be no differences in auditory cortices across different degrees of syncopation, but that there would be greater entrainment in PMC for moderate degrees of syncopation.

\section{Methods}

\section{Participants}

Thirty-nine participants with self-reported normal hearing were recruited from the Ryerson University psychology student participant pool. Based on selfreport, nine were left-handed, 11 had perfect pitch, 15 considered themselves musical, and 13 were musically active at the time of testing (playing or singing in a group or on their own). They had an average of 4 years of musical experience with a range of 0 to 15 years. This study was approved by the Ryerson Research Ethics Board and all participants completed informed consent prior to the study. A power analysis based on the inverted-U results of Witek et al. (2014) with 
power fixed at .8 revealed that 17 participants would be necessary for this study. Because this study was run in conjunction with Study 3 (which required 34 participants), it is possible that this study is overpowered. The calculated power of the results from the same inverted-U analysis in this study was 0.99 .

\section{Stimuli}

Stimuli ${ }^{15}$ consisted of six different rhythms, two each with low, moderate, and high degrees of syncopation (Table 1). The two moderately-syncopated rhythms were the Son and Rumba clave rhythms and were matched for number of note onsets. The low and high syncopation degree rhythms consisted of modified versions of the Son and Rumba clave rhythms that were either more "on-beat" or more "off-beat", respectively. The syncopation of each rhythm was quantified using the syncopation index described by Longuet-Higgins and Lee (1984; Witek et al., 2014). The syncopation index is calculated by first assigning a weight to each note in a rhythm based on its position in the metrical hierarchy. The beat level is assigned a value of 0 , a subdivision by 2 is assigned a value of -1 , and so on. If the weight of a note is greater than the weight of the preceding note, then the pair of notes is said to constitute a syncopation, with a value equal to the difference between the two weights. The syncopation index of a rhythm is taken as the sum of syncopation values for all pairs of notes.

The rhythms were generated using Cubase 9.0 and were played using a piano timbre from the HALion Sonic SE sample library, playing a chord typically used in jazz (Dsus13). The beat frequency was also outlined during each excerpt with an isochronous hi-hat sound, to ensure that participants would perceive the rhythm with the intended meter. The tempo of each stimulus presentation was randomized to be $1.5,1.6,1.7,1.8$, or $1.9 \mathrm{~Hz}(90,96,102,108$, or 114 beats per minute). This was done to avoid carry-over effects between trials, where entrainment on a given trial might be affected by entrainment to the same tempo on the previous trial. Regardless of the tempo, each rhythm was

\footnotetext{
${ }^{15}$ Stimuli were obtained from Tomas Matthews and Maria Witek
} 
played for 30 seconds.

Table 1. List of stimuli (Study 2)

\begin{tabular}{llr}
\hline & Syncopation & Syncopation \\
Rhythm & Degree & Index \\
\hline Low 3 & low & 0 \\
Low 6 & low & 4 \\
Son Clave 3-2 & moderate & 5 \\
Rumba Clave & moderate & \\
3-2 & & 18 \\
High 1 & high & 16 \\
High 3 & high & \\
\hline
\end{tabular}

\section{Procedure}

Participants were seated at a computer in a sound-attenuated chamber. Stimulus presentation was controlled using MATLAB (version R2017a; The Mathworks, Inc.), and excerpts were played over Logitech Z130 loudspeakers in two counterbalanced blocks. In one block, participants were asked to simply listen to each excerpt and to refrain from moving. In the other block, participants were asked to tap along to the beat on a MIDI drum pad with whichever hand they felt more comfortable. If the participant was unsure about how to do this, it was demonstrated to them by the experimenter. In each block, each of the six rhythms was played 5 times (once for each tempo) in random order, for a total of 30 trials. Stimulus presentation was self-paced, and after each trial participants were asked to answer the question "To what extent does this rhythm make you want to move?" on a 7-point Likert scale.

Stimulus presentation was controlled with MATLAB (version R2017a; The Mathworks, Inc.) running on a Windows computer. EEG data were recorded on a separate Windows computer using a 128-channel BioSemi ActiveTwo sys- 
tem and were digitized at $512 \mathrm{~Hz}$. Tapping data were recorded using a Roland HPD-10 drum pad and Pro Tools 12.8.0 on a Macintosh computer running OSX 10.11.6. Analyses were carried out using MATLAB (version R2017a; The Mathworks, Inc.) on a Macintosh computer running OSX 10.12.6, and included custom scripts, the EEGLAB Toolbox (version 14.1.1; Delorme \& Makeig, 2004), and MIDI Toolbox (Toiviainen \& Eerola, 2016). Statistical analyses were carried out in R (R Core Team, 2017). All task, analysis, and statistical scripts are available online (https://github.com/gabenespoli/entrainment).

EEG Analysis. EEG data were preprocessed in two pipelines with different high-pass filters in order to improve the quality of the ICA (Debener et al., 2010; I. Winkler et al., 2015). For the first pipeline, raw EEG data were high-pass filtered at $1 \mathrm{~Hz}$ using the EEGLAB function pop_eegfiltnew. Bad channels were identified using the clean_rawdata plugin. This plugin works by first windowing the data into 1-second non-overlapping windows. In each window, each channel is interpolated based on all other channels, and this interpolation is correlated with the actual data from that channel. If this correlation is lower than 0.8, the channel is flagged for the given window. Any channel with flags in more than half of the windows is rejected. Data were then rereferenced to the average, and line noise was removed (pop_cleanline). Sections of data where no music was presented (i.e., while entering responses on the computer) were removed (pop_epoch), and the remaining data were subjected to the extended version of the Infomax ICA (Bell \& Sejnowski, 1995) as implemented in EEGLAB (pop_runica).

In the second pipeline, raw EEG data were high-pass filtered at $0.5 \mathrm{~Hz}$. This cutoff was chosen to be as high as possible without cutting off frequencies of interest (i.e., groupings of two at a tempo of $1.5 \mathrm{~Hz}$ would be $0.75 \mathrm{~Hz}$ ). Channels that were identified as bad in the first pipeline were removed. All other channels were referenced to the average and epoched. Next, the ICA weights and sphering matrices (i.e., EEG.icaweights and EEG.icasphere) obtained from the first pipeline were used with the electrode data from the second pipeline to compute IC activity. 
Regions of interest were defined based on Brodmann areas (BA): the PMC region comprised BA6, and the auditory cortex region comprised BA22, BA41, and BA42. In order to localize components with respect to these target brain regions, equivalent dipole models were fit to each IC (pop_dipfit). For each region of interest, an IC was selected if its Talairach coordinates were within 8.66 $\mathrm{mm}^{16}$ of the target brain region (Lancaster et al., 1997, 2000) and the residual variance of its corresponding dipole model was less than $15 \%$. If a particular IC was within $5 \mathrm{~mm}$ of both regions it was assigned to the closer region; if it was the same distance from both it was assigned to both. The average location of selected ICs is plotted in Figure 6.

Entrainment of each resulting IC was determined in the following way. First, each IC was subjected to an FFT with a bin width of $0.0078 \mathrm{~Hz}$. This bin width allowed for averaging across a maximum of 5 bins centered on the target frequency before it would begin to overlap with the neighbouring tempo. The noise floor was removed by subtracting activity in the surrounding bins as in Nozaradan et al. (2011). The extent of entrainment was defined as the mean value of three bins centered on the target frequencies. Target frequencies were meter-related, all having simple-integer ratio relationships with the beat rate. They included one half of the beat rate (i.e., half-note level; grouping beats in twos), the beat rate (quarter-note level), subdivision by two (eighth-note level) and by 4 (sixteenth-note level). Entrainment values were then normalized for each participant by dividing each value by the maximum value for that participant.

Tapping Analysis. MIDI files were exported from Pro Tools and imported into MATLAB using MIDI Toolbox (Toiviainen \& Eerola, 2016). The onset times of each tap were used to calculate a mean onset asynchrony for each trial in the following way. First, each onset was matched to the beat time to which it was closest. Then, the onset asynchrony was calculated as the difference

\footnotetext{
${ }^{16}$ The localization procedure creates a 3D cube centered on the given Talairach coordinates. The cube extends $5 \mathrm{~mm}$ on all sides resulting in a total size of $11 \times 11 \times 11 \mathrm{~mm}$. The furthest distance from the center of the cube to the edge (i.e., the corner of the cube) is $8.66 \mathrm{~mm}$.
} 

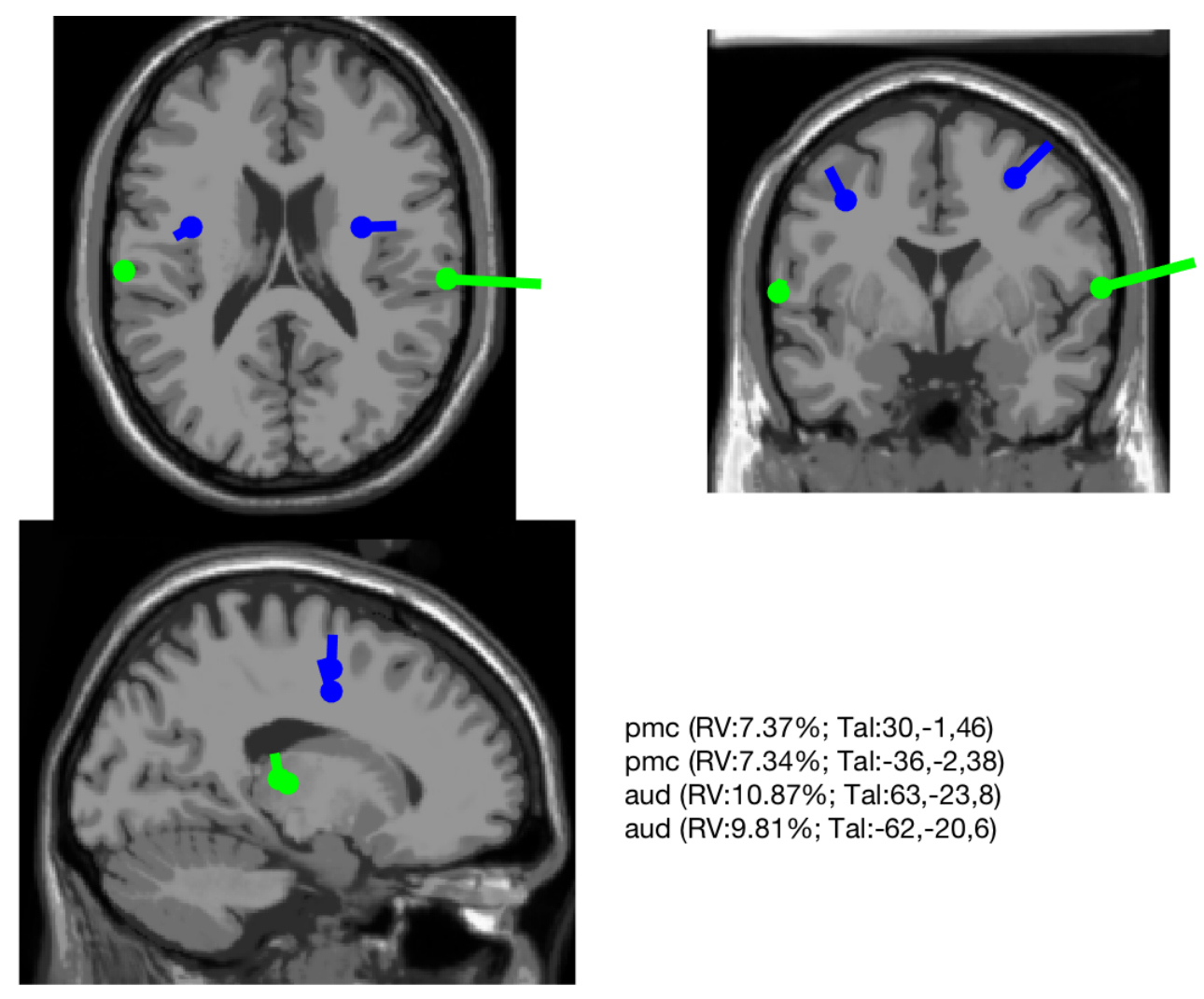

pmc (RV:7.37\%; Tal:30,-1,46)

pmc (RV:7.34\%; Tal:-36,-2,38)

aud (RV:10.87\%; Tal:63,-23,8)

aud (RV:9.81\%; Tal:-62,-20,6)

Figure 6. Talairach coordinates of selected ICs. Talairach coordinates for selected ICs were sorted based on hemisphere and region, averaged across participants, and plotted onto standardized MRI images. dark blue = premotor ICs; light green = auditory ICs; pmc = premotor cortex; aud = auditory cortex; RV $=$ residual variance; $\mathrm{Tal}=$ Talairach; Line indicates the direction of the dipole. 
between each onset and its matched beat time. The standard deviation of these differences was used as the measure of tapping variability.

\section{Results}

Analyses were run separately for ratings of wanting to move, tapping variability, and neural entrainment. For each dependent variable, an ANOVA was performed with Syncopation Degree (low, moderate, high) as a within-subjects variable.

To test the hypothesis that moderately-syncopated rhythms elicit greater feelings of groove than both low and high, an inverted-U analysis was also performed (Witek et al., 2014) to test if a parabola would be a better fit to the data than a linear model. For this analysis, each participant's data were regressed against the stimuli with the Syncopation Index (Longuet-Higgins \& Lee, 1984) as the predictor. Both linear and quadratic models were fit, and a one-way ANOVA of the adjusted $\mathrm{R}^{2}$ values was performed with Model (linear, quadratic) as the within-subjects factor.

\section{Ratings of Wanting to Move}

A one-way ANOVA on participants' ratings of wanting to move with Syncopation Degree (low, moderate, high) as the within-subjects factor revealed a main effect of Syncopation Degree $(F(2,76)=129.2, p<.001)$. Post-hoc $t$-tests revealed that all degrees of syncopation were different from one another, with moderate getting the highest ratings (mean $=4.9$; mean of low $=4.6$; mean of high $=2.4$; Figure 7).

The inverted- $\mathrm{U}$ analysis revealed a main effect of Model $(F(1,38)=19.5, p$ $<.001)$, where the quadratic model had higher $\mathrm{R}_{\text {adj }}^{2}$ values $(0.728)$ than the linear model (0.654). This replicates Witek et al. (2014) who also found that a quadratic model was a better fit for ratings of wanting to move when regressed 
against Longuet-Higgins and Lee's (Longuet-Higgins \& Lee, 1984) syncopation index.

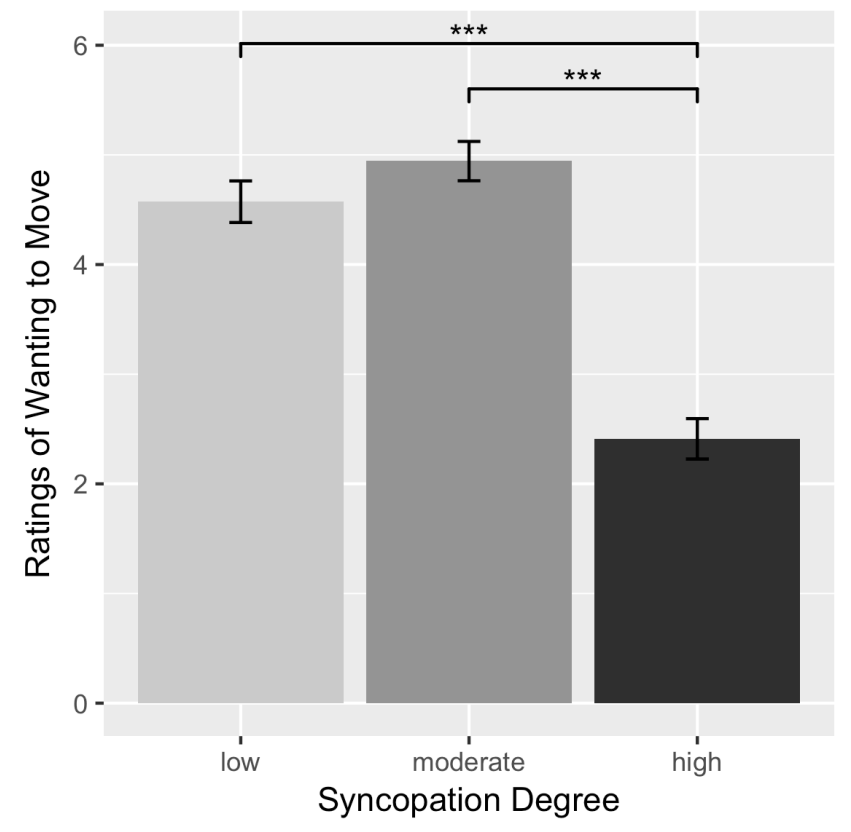

Figure 7. Ratings of wanting to move. Participants' ratings of wanting to move were significantly higher for moderately-syncopated rhythms. Error bars represent standard error. Asterisks represent $p$-values less than .001 (***)

\section{Tapping Variability}

A one-way ANOVA was run on participants' tapping variability with the withinsubjects factor of Syncopation Degree (low, moderate, high). Tapping variability was defined as the standard deviation of the onset asynchrony. There was a main effect of Syncopation Degree $(F(2,74)=26.29, p<.001)$. Post-hoc $t$-tests revealed that participants were more variable when tapping to rhythms with high degrees of syncopation $($ mean $=0.16)$ than to rhythms with low $($ mean $=$ $0.089 ; t(44.1)=-5.941, p<.001)$ or moderate $($ mean $=0.099 ; t(45)=-5.262$, $p<.001$ ) degrees of syncopation (Figure 8 ). The inverted-U analysis did not reveal a significant difference between linear and quadratic modeling. 


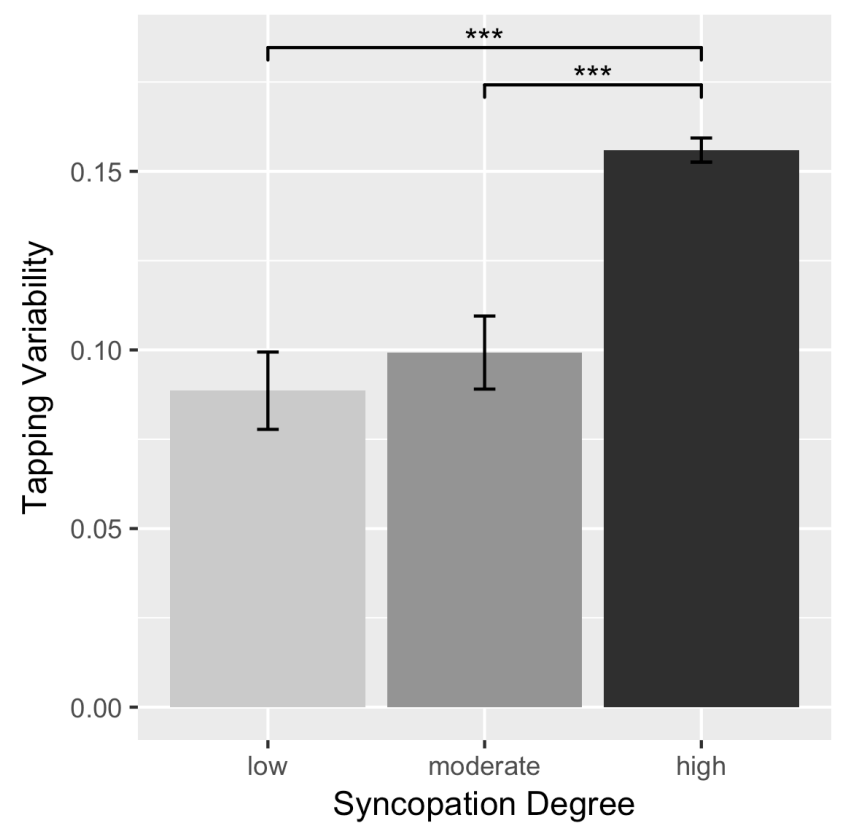

Figure 8. Tapping variability. Participants were more variable when tapping along to rhythms with high degrees of syncopation than low or moderate degrees of syncopation. Error bars represent standard error. Asterisks represent $p$ values less than .001 (***) 


\section{Neural Entrainment}

An omnibus ANOVA was run with Syncopation Degree (low, moderate, high), Metric Level (half-, quarter-, eighth-, and sixteenth-note levels), and Brain Region (auditory, PMC) as within-subjects factors. There were significant main effects of Syncopation Degree $(F(2,76)=5.903, p=.0041)$ and Metric Level $(F(3,114)=122.2, p<.001)$. There was also a significant interaction between Syncopation Degree and Metric Level $(F(6,228)=2.818, p=.011)$. There were no main effects $(F(1,38)=0.8831, p=.35)$ or interactions involving Brain Region. As a result, entrainment values for the different regions were averaged together for the rest of the analyses.

For Syncopation Degree, post-hoc $t$-tests did not reveal any significant differences between levels (Figure 9). This is likely due to the significant interaction, reported below. The inverted-U analysis did not reveal a significant difference between linear and quadratic modeling.

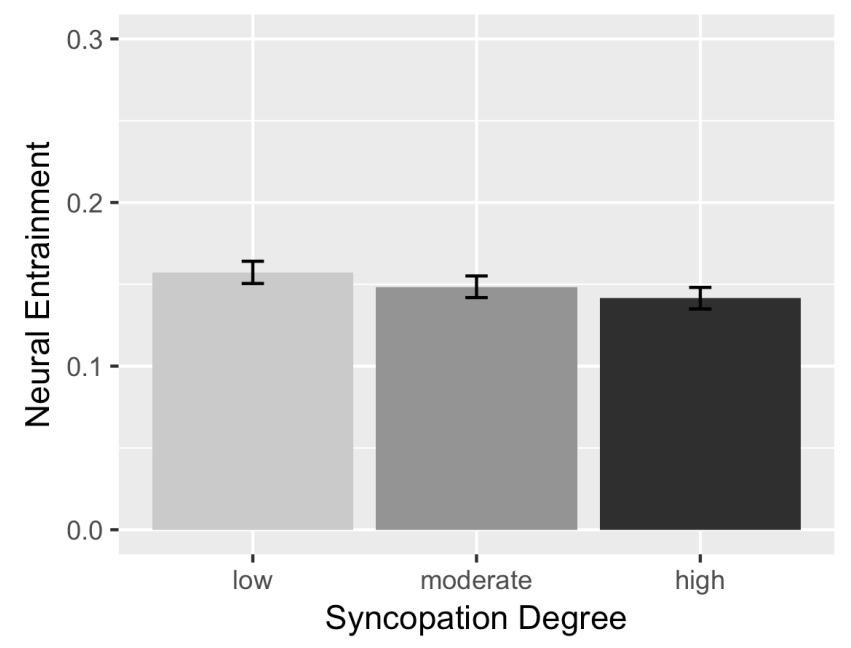

Figure 9. Neural entrainment by syncopation degree. Error bars represent standard error.

For Metric Level, it was found that all levels were significantly different from one another (all $p$-values were less than .001; Figure 10). 


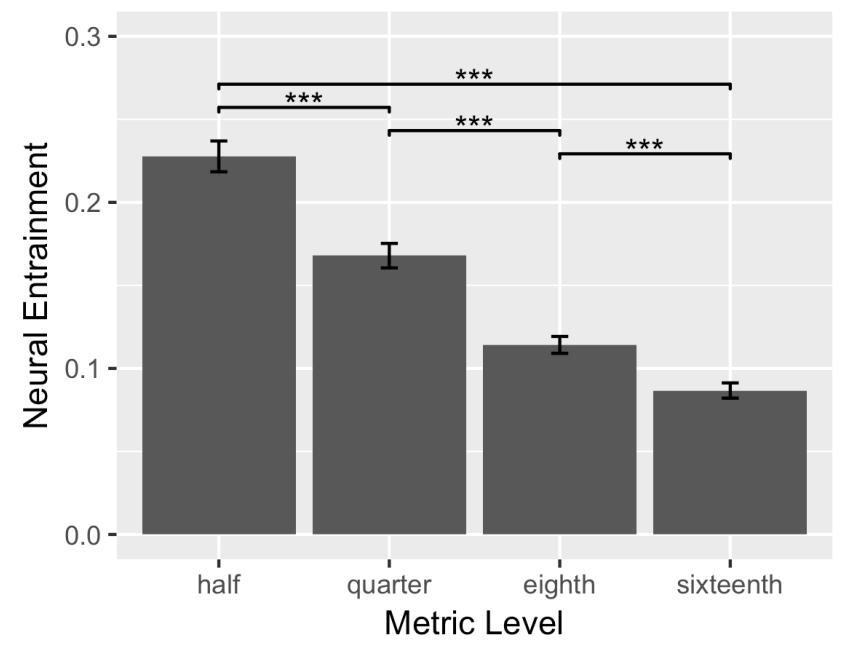

Figure 10. Neural entrainment by metric level. Error bars represent standard error. Asterisks represent $p$-values less than .001 (***)

To investigate the interaction, $t$-tests were performed between the levels of Syncopation Degree separately for each Metric Level (Figure 11). For the quarternote (beat) level, moderate degrees of syncopation $($ mean $=0.153)$ elicited less neural entrainment than low (mean $=0.185 ; t(72.4)=1.658, p=.1$ ), with marginal significance. For the eighth-note level, low degrees of syncopation $($ mean $=0.129)$ elicited significantly greater neural entrainment than high (mean $=0.100 ; t(67)=2.28, p=.026)$.

The inverted-U analysis was carried out for each metric level separately, but none revealed a significant difference between linear and quadratic modeling.

\section{Discussion}

For ratings of wanting to move, moderate levels of syncopation elicited the highest ratings, which replicates previous work (Sioros et al., 2014; Witek et al., 2014). Unlike rhythms with low and high degrees of syncopation, the brain's attempt to model the rhythm is associated with some prediction error. This prompts the system to reconcile the differences between the model and the input 


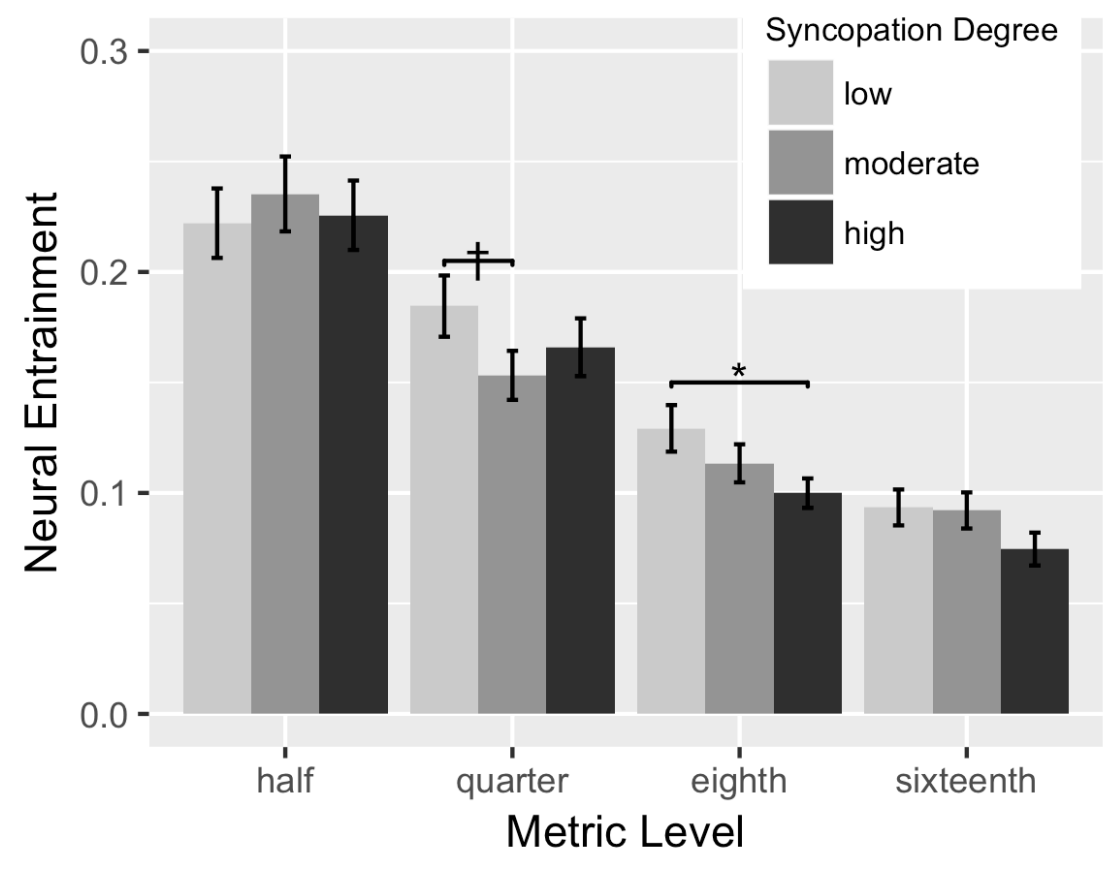

Figure 11. Neural entrainment by metric level and syncopation degree. Error bars represent standard error. Asterisks represent $p$-values less than .05 $\left(^{*}\right)$ and $.1(\dagger)$. 
by reinforcing the model. If the model resides in premotor areas of the brain, then a desire to reinforce the model could involve a greater desire to move.

Ratings of wanting to move were also higher for low compared to high degrees of syncopation. While this was not expected, it can be interpreted with regard to the predictive coding framework. At low degrees of syncopation, the brain is able to draw on a reliable model of the input that it can use to predict the location of upcoming beats (as evidenced by the low tapping variability to these rhythms). At high degrees of syncopation, there is no such reliable model. The higher ratings for low degrees of syncopation might suggest that the model resides in motor areas, and that hearing the rhythm elicits some neural resonance there that gives rise to an increased desire to move. And since there is no prediction error, this desire is less than for moderately-syncopated rhythms.

For tapping performance, variability was worse for highly syncopated rhythms and better for both low and moderate degrees of syncopation, which is in line with the hypotheses based on predictive coding. Rhythms for which the brain is able to draw on an existing model elicit the best tapping performance, because a reliable prediction can be made about the temporal location of upcoming beats. For rhythms which are too complex, the brain does not have an existing model and cannot make good predictions.

For neural entrainment it was predicted that auditory and premotor regions would have different patterns of response across different degrees of syncopation, but this was not the case. Both regions seemed to exhibit greater entrainment for low versus high degrees of syncopation. One possible explanation is that higher entrainment is related to how well the brain's model fits the input. Lower degrees of syncopation are relatively easier to model, and thus give rise to greater entrainment.

The significant interaction of syncopation degree with metric level was particularly revealing and tells a more complicated story. At the beat level, neural entrainment seems to be suppressed for moderate degrees of syncopation. This was unexpected; why does entrainment at the beat level go away when there is 
some prediction error (and when feelings of wanting to move are highest)? There are at least two potential explanations for this pattern. First, participants were instructed not to move while listening. Suppressing overt movement at least means preventing neural firing that would cause muscles to contract. If this neural firing comprises a model in PMC, then activation of this model should be suppressed as well. Since the model likely consisted of the beat frequency, this resulted in less neural entrainment at that frequency. In a sense, participants were told that their internal model for moderate degrees of syncopation should not be reinforced. This finding speaks to the embodied nature of groove, suggesting that activating a premotor model, desiring to move, and actually moving are very much integrated with one another when it comes to perceiving certain rhythms. When perceiving a rhythm that defies the expectancies of a meter, the brain attempts to understand the rhythm by updating and reinforcing its internal model of the meter. This would involve strengthening relevant motor plans, perhaps by executing them by moving.

Another possible explanation has to do with the mechanics of updating an internal model. Greater neural entrainment means that many neurons are synchronously firing at a specific frequency. If this synchronous firing is considered the "model", and this model is being activated (i.e., is firing), you would expect to see greater neural entrainment at that frequency. This would be the expected result if the model matched the input very well (e.g., for low degrees of syncopation). However, if this model is a close-but-not-perfect match for the input, and is in the process of being "updated", it seems possible that there would be some desynchronization at this frequency as the brain attempts to reorganize the model. This mechanistic account focusing on destructive interference of endogenous rhythms is reminiscent of the manner in which $m u$-band desynchronization has been interpreted under movement observation conditions (McGarry et al., 2012).

The pattern of neural entrainment at the eighth-note level, however, seems to behave more similarly to participants' tapping performance, where lower degrees of syncopation elicited greater entrainment. Subdivisions of the beat 
are represented at the eighth-note level (for the low-syncopation stimuli), and subdividing the beat has been found to reduce tapping variability (Repp, 2010). So this enhanced entrainment at the eighth note level may be related to accuracy of movement synchronization.

It was also found that neural entrainment decreases with increasing metric level, where metric levels representing larger groups of notes elicit greater entrainment than subdivisions. It is established that the power of the EEG signal tends to display this $1 / f$ behaviour, where the magnitude of spectral activity decreases with increasing frequency (Demanuele et al., 2007), so in some sense this is not unexpected. However, if different populations of neurons are synchronizing their firing to the different metrical levels present in the stimulus, then points of metrical accent would likely involve more neurons firing and greater EEG activity at that frequency.

\section{Conclusions}

The current study replicates previous work where moderate degrees of syncopation elicit greater feelings of wanting to move (Sioros et al., 2014; Witek et al., 2014). It also finds that tapping variability is lower for both low and moderate degrees of syncopation. This is interpreted as the brain being able to use an existing, suitable model for predicting the auditory input. Neural entrainment was not found to be greater in PMC for moderate degrees of syncopation. Instead, entrainment at the beat frequency was found to be lower. It is suggested that this is either because a) participants were instructed to refrain from moving, and are therefore suppressing the reinforcement of the model and the desire to move, or b) the model is being updated, and this is associated with a desynchronization of activity. 
Study 3: Acoustic Aspects of Music

What are the physical properties of a sound signal that would drive entrainment of PMC neurons? There are at least two areas of work relating to neural entrainment that investigate acoustic features of music that could help select relevant features: subjective evaluation of groove and sensorimotor synchronization studies. Both of these literatures have identified important acoustic features, and are thus a useful place to draw insight as to what features might be relevant to PMC entrainment.

A sense of groove is traditionally defined as music that creates a desire to move in a listener (Madison, 2006), and has been linked to increased neural excitability of the motor system (Stupacher et al., 2013). Participants' ratings of groove have been found to correlate with certain acoustic features (Madison et al., 2011; Stupacher et al., 2016); among these are beat salience, event variability, RMS variability, and low-frequency spectral flux.

Beat salience (Madison et al., 2011) or pulse clarity (Lartillot, Eerola, Toiviainen, \& Fornari, 2008) is a feature indexing how easily a listener can perceive the underlying rhythmic pulse of a piece of music. This feature is calculated by first applying an onset detection function, such that subsequent analyses can focus on note onsets instead of the whole acoustic signal. This is usually accomplished by filtering the signal with a bank of non-overlapping filters, rectifying, and down-sampling. The resulting onset velocity curve is a time-varying signal that contains peaks at purported note onsets. To calculate beat salience, the onset signal is subjected to an autocorrelation function to assess the extent to which it repeats itself. By focusing on the autocorrelation at lags that are related to the beat frequency, an estimate can be obtained for the extent to which the signal emphasizes the underlying metric structure.

The strength of the underlying pulse has been associated with both ratings of groove and movement synchronization with music. In a study investigating the phenomenology of groove, participants noted that a sense of groove depends on 
a strong underlying beat (Janata et al., 2012). Songs with drums and bassinstruments that tend to define rhythmic structure in an unequivocal mannertend to elicit higher groove ratings from participants (Stupacher et al., 2016). Beat salience and pulse clarity have also been found to correlate with groove ratings (Madison et al., 2011).

Event variability (Madison et al., 2011) is also determined by first establishing the onset velocity curve of the signal. Then, the variance of this curve is computed per beat. This metric is considered a convenient proxy for the variability of notes at the sub-beat level, and is sensitive to the fact that relative onset amplitudes may differ across time. ${ }^{17}$ Event variability has been found to be a good predictor of participants' groove ratings (Madison et al., 2011; Stupacher et al., 2016).

Root mean square (RMS) variability is a feature relating to the variability in the intensity and loudness of the signal. It is calculated by first squaring all values in the signal, taking a sliding window average, and finally taking the square root. The standard deviation of the resulting time-varying RMS waveform is then computed as a measure of variability. While RMS variability has been found to correlate with groove ratings, RMS has not (Stupacher et al., 2016).

Spectral flux is a measure of moment-to-moment differences in the spectrum of a sound signal, and is often calculated separately for different frequency bands. Flux in low-frequency bands (i.e., between 0 and $200 \mathrm{~Hz}$ ) has been correlated with groove ratings (Stupacher et al., 2016). The authors also note that the songs which received the highest groove ratings tended to have drums, including a (low frequency) kick drum. ERP work has also suggested that lower frequencies are associated with superior timing perception in music (Hove, Marie, Bruce, \& Trainor, 2014). Stupacher et al. (2016) experimentally manipulated

\footnotetext{
${ }^{17}$ Event variability is called "event density" in Madison et al:s (2011) original article. "Event variability" is used here instead because a) it can be distinguished from Lartillot and Toiviainen's (2007) event density, where peaks in the onset velocity curve are simply counted per unit time, and b) it better-characterizes what is actually being described in the signal. In a study comparing the two features, event variability, but not even density, was found to predict participants' groove ratings (Stupacher et al., 2016).
} 
the frequency of the bass drum in their stimuli, and found that lower frequencies were associated with higher groove ratings.

The ability to tap along with a rhythm has been linked to the extent of neural entrainment to the beat frequency (Nozaradan et al., 2015). Strong endogenous neural entrainment has also been found to correlate with tapping performance and rhythmic prediction abilities (Nozaradan et al., 2016). Thus, acoustic features that aid in sensorimotor synchronization might also drive neural entrainment.

Work on acoustic features and movement have implicated a similar set of features. Burger et al. (2012) used motion capture to investigate movement regularity when participants were asked to move along with music. They found that the regularity of the underlying pulse (pulse clarity; Lartillot et al., 2008) and low-frequency flux (i.e., between 0 and $200 \mathrm{~Hz}$ ) was significantly correlated with movement regularity. Lower frequencies have also been associated with less variable and more forceful tapping (Stupacher et al., 2016).

Percussiveness is another feature that has been related to movement regularity (Burger et al., 2012, 2013). This feature is calculated as the average slope of the onsets of each note event (mirattackslope in MIR Toolbox). A steeper average slope would mean shorter onset for each note, which may in turn facilitate synchronization. Interestingly, percussiveness has been associated with synchronization of specific body parts such as the head and hands, whereas other features (like pulse clarity) were associated with movement of the whole body (Burger et al., 2013).

The purpose of Study 3 was to identify acoustic features that could predict PMC entrainment at the beat frequency. Participants listened to drum rhythms and a) rated them on the extent to which it made them want to move, b) tapped along, and c) passively listened while EEG was recorded. To achieve this, three different approaches were taken. First, a multiple linear regression model was used to model the rating data using acoustic features that have been previously implicated. This model was then applied to the neural entrainment 
data to assess how well these features would predict entrainment. Next, the same procedure was carried out on the tapping data, using a slightly different set of features that have been implicated in the tapping literature. Finally, an exploratory analysis was done, whereby all previously discussed features were entered into a stepwise regression as a means of variable selection.

\section{Methods}

\section{Participants}

Thirty-nine participants with self-reported normal hearing were recruited from the Ryerson University psychology student participant pool. Based on selfreport, nine were left-handed, 11 had perfect pitch, 15 considered themselves musical, and 13 were musically active at the time of testing (playing or singing in a group or on their own). They had an average of 4 years of musical experience with a range of 0 to 15 years. This study was approved by the Ryerson Research Ethics Board and all participants completed informed consent prior to the study. A power analysis based on correlation analyses from previous studies (Burger et al., 2012; Stupacher et al., 2016) revealed a large effect size $\left(f^{2}=.60\right)$, indicating that a sample size of 34 would be necessary.

\section{Stimuli}

Stimuli consisted of drumbeats that were created in Garageband (version 10.2.0 on macOS Sierra 10.12.6) using the virtual drummer interface. This interface uses sliders to modify the intensity and complexity of default drum patterns. A total of thirty rhythms were created, each using a different default pattern, and were modified to achieve a relatively wide range of pulse clarity and beat salience. Rhythms were restricted to use only the kick drum, snare drum, and closed hi-hat (MIDI notes $\mathrm{C} 1, \mathrm{D} 1$, and F\#1, respectively), and were quantized to the 64 th note grid. Each rhythm was exported as a .wav file at five different 
tempos (90, 96, 102, 108, and $114 \mathrm{bpm}$; 1.5, 1.6, 1.7, 1.8, and $1.9 \mathrm{~Hz})$ using the "Deep Tech" electronic drum kit in Garageband. Regardless of the tempo, each rhythm was played for 30 seconds. This resulted in a total of 150 stimulus tokens.

Acoustic features were extracted from all stimuli using MATLAB R2017a (The Mathworks, Inc.). Beat salience and event variability were extracted using the ShakeIt Rhythm Descriptors code obtained from Madison et al. (2011). MIR Toolbox (Lartillot \& Toiviainen, 2007) was used to extract the remaining features: pulse clarity (mirpulseclarity function), RMS variability (mirrms, then compute standard deviation), percussiveness (mirattackslope), and subband flux (mirfilterbank and mirflux) in the following bands: 0-50 Hz, 50-100 Hz, 100-200 Hz, 200-400 Hz, 400-800 Hz, 800-1600 Hz, 1600-3200 Hz, 3200-6400 $\mathrm{Hz}, 6400-12800 \mathrm{~Hz}$, and 12800-22050 Hz.

\section{Procedure}

Participants were seated at a computer in a sound-attenuated chamber. Stimulus presentation was controlled using MATLAB (version R2017a; The Mathworks, Inc.), and excerpts were played over Logitech Z130 loudspeakers in two blocks. In one block, participants were asked to simply listen to each excerpt and to refrain from moving. In the other block, participants were asked to tap along to the beat on a MIDI drum pad with whichever hand they felt more comfortable. If the participant was unsure about how to do this, it was demonstrated to them by the experimenter. In each block, each of the 30 drum patterns were played in random order. The tempo of each trial was random, but the same drum pattern-tempo pairings were used for both blocks. Stimulus presentation was self-paced, and after each trial participants were asked to answer the question "To what extent does this rhythm make you want to move?" on a 7-point Likert scale.

Stimulus presentation was controlled with MATLAB (version R2017a; The Mathworks, Inc.) running on a Windows computer. EEG data were recorded 
on a separate Windows computer using a 128-channel BioSemi ActiveTwo system and were digitized at $512 \mathrm{~Hz}$. Tapping data were recorded using a Roland HPD-10 drum pad and Pro Tools 12.8 .0 on a Macintosh computer running OSX 10.11.6. Analyses were carried out using MATLAB (version R2017a; The Mathworks, Inc.) on a Macintosh computer running OSX 10.12.6, and included custom scripts, the EEGLAB Toolbox (version 14.1.1; Delorme \& Makeig, 2004), and MIDI Toolbox (Toiviainen \& Eerola, 2016). Statistical analyses were carried out in $\mathrm{R}$ ( $\mathrm{R}$ Core Team, 2017). All task, analysis, and statistical scripts are available online https://github.com/gabenespoli/entrainment.

EEG Analysis. EEG data were preprocessed in two pipelines with different high-pass filters in order to improve the quality of the ICA (Debener et al., 2010; I. Winkler et al., 2015). For the first pipeline, raw EEG data were high-pass filtered at $1 \mathrm{~Hz}$ using the EEGLAB function pop_eegfiltnew. Bad channels were identified using the clean_rawdata plugin. This plugin works by first windowing the data into 1-second non-overlapping windows. In each window, each channel is interpolated based on all other channels, and this interpolation is correlated with the actual data from that channel. If this correlation is lower than 0.8, the channel is flagged for the given window. Any channel with flags in more than half of the windows is rejected. Data were then rereferenced to the average, and line noise was removed (pop_cleanline). Sections of data where no music was presented (i.e., while entering responses on the computer) were removed (pop_epoch), and the remaining data were subjected to the extended version of the Infomax ICA (Bell \& Sejnowski, 1995) as implemented in EEGLAB (pop_runica).

In the second pipeline, raw EEG data were high-pass filtered at $0.5 \mathrm{~Hz}$. Channels that were identified as bad in the first pipeline were removed. All other channels were referenced to the average and epoched. Next, the ICA weights and sphering matrices (i.e., EEG.icaweights and EEG.icasphere) obtained from the first pipeline were used with the electrode data from the second pipeline to compute IC activity. 
Regions of interest were defined based on Brodmann areas (BA): the PMC region comprised BA6, and the auditory cortex region comprised BA22, BA41, and BA42. In order to localize components with respect to these target brain regions, equivalent dipole models were fit to each IC (pop_dipfit). For each region of interest, an IC was selected if its Talairach coordinates were within 8.66 $\mathrm{mm}^{18}$ of the target brain region (Lancaster et al., 1997, 2000) and the residual variance of its corresponding dipole model was less than $15 \%$. If a particular IC was within $5 \mathrm{~mm}$ of both regions it was assigned to the closer region; if it was the same distance from both it was assigned to both. The average location of selected ICs is plotted in Figure 12.

Entrainment of each resulting IC was determined in the following way. First, each IC was subjected to an FFT with a bin width of $0.0078 \mathrm{~Hz}$. This bin width allowed for averaging across a maximum of 5 bins centered on the target frequency before it would begin to overlap with the neighbouring tempo. The noise floor was removed by subtracting activity in the surrounding bins as in Nozaradan et al. (2011). The extent of entrainment was defined as the mean value of three bins centered on the target frequencies. Target frequencies were meter-related, and all had simple-integer ratio relationships to the beat rate. They included one half of the beat rate (i.e., half-note level; grouping beats in twos), the beat rate (quarter-note level), subdivision by two (eighth-note level) and by 4 (sixteenth-note level). Entrainment values were then normalized by dividing each value by the maximum value for that participant.

Tapping Analysis. MIDI files were exported from Pro Tools and imported into MATLAB using MIDI Toolbox (Toiviainen \& Eerola, 2016). This returns an onset time, a duration, and an intensity for each tap. For each trial, average duration and average intensity of taps was obtained by averaging the respective values for all taps on that trial. The onset times were used to calculate a mean onset asynchrony for each trial in the following way. First, each onset was

\footnotetext{
${ }^{18}$ The localization procedure creates a $3 \mathrm{D}$ cube centered on the given Talairach coordinates. The cube extends $5 \mathrm{~mm}$ on all sides resulting in a total size of $11 \times 11 \times 11 \mathrm{~mm}$. The furthest distance from the center of the cube to the edge (i.e., the corner of the cube) is $8.66 \mathrm{~mm}$.
} 

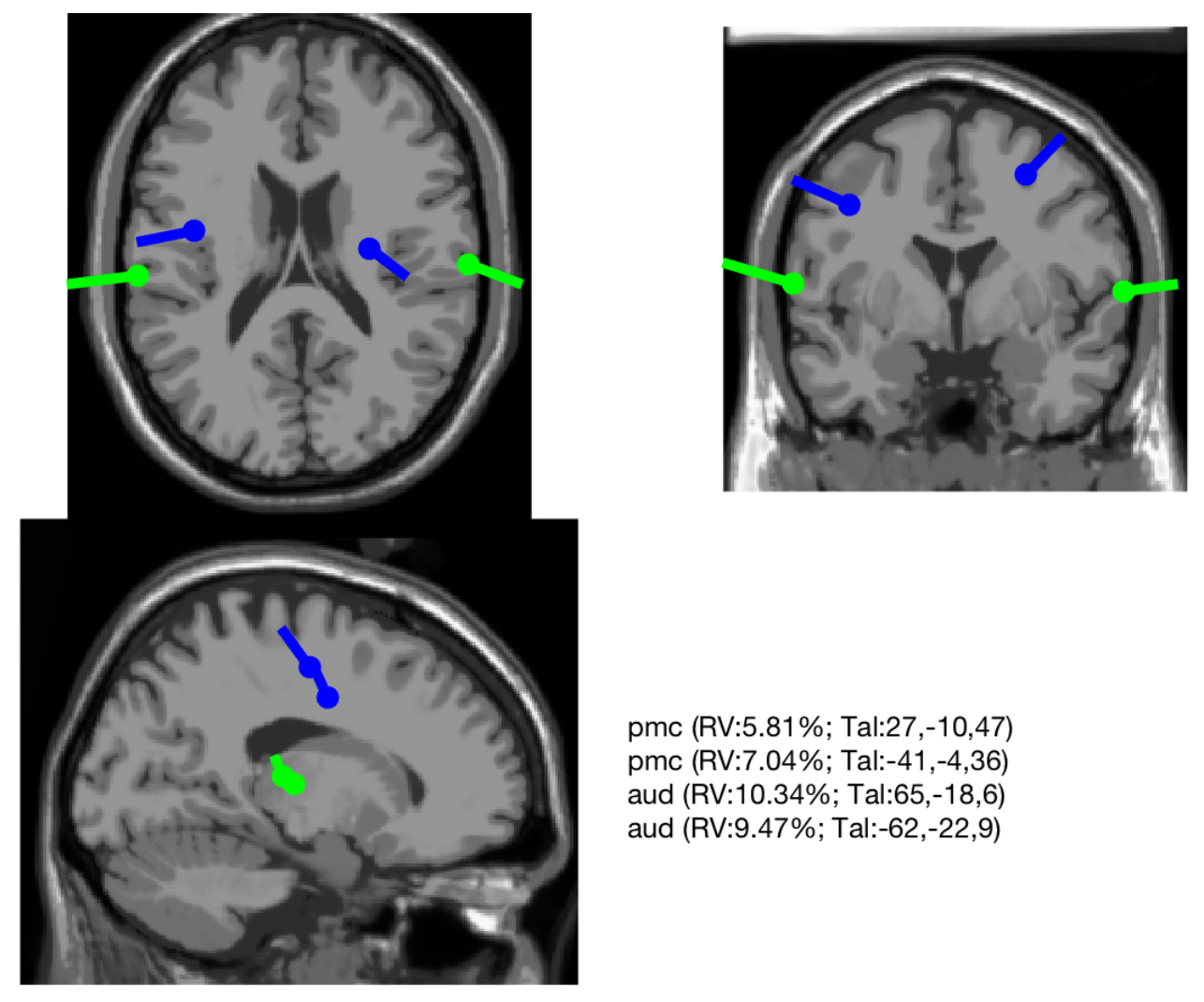

pmc (RV:5.81\%; Tal:27,-10,47)

pmc (RV:7.04\%; Tal:-41,-4,36)

aud (RV:10.34\%; Tal:65,-18,6)

aud (RV:9.47\%; Tal:-62,-22,9)

Figure 12. Talairach coordinates of selected ICs. Talairach coordinates for selected ICs were sorted based on hemisphere and region, averaged across participants, and plotted onto standardized MRI images. dark blue $=$ premotor ICs; light green $=$ auditory ICs; $\mathrm{pmc}=$ premotor cortex; aud $=$ auditory cortex; $\mathrm{RV}=$ residual variance; Tal $=$ Talairach; Line indicates the direction of the dipole. 
matched to the beat time to which it was closest. Then, the absolute difference between each onset and its matched beat time was calculated, and the average of these differences was considered the mean onset asynchrony.

Feature Selection. While a number of acoustic features have been found to relate to groove ratings and movement regularity, there is no such literature that exists for neural entrainment. To identify acoustic features that might be related to PMC entrainment, the following two methods were used. First, features previously found to correlate with groove ratings or tapping performance were selected for the two models. To avoid multicollinearity, a feature was removed if a) it was significantly correlated with another feature with a coefficient greater than .7, and b) it was less correlated with the dependent measure than the other feature. The resulting features were then used in a multiple linear regression model to predict Ratings of Wanting to Move and Tapping Variability, respectively. These same two models were then used to predict PMC entrainment. Second, a more exploratory approach was taken by selecting features via correlation with PMC Entrainment. All of the previously-mentioned features that were highly-correlated with PMC Entrainment were entered into a multiple linear regression model.

\section{Results}

\section{Model of Ratings of Wanting to Move}

Feature selection. Acoustic features that have been identified in previous work to be related to groove ratings (Madison et al., 2011; Stupacher et al., 2016) were selected for use in the model: beat salience, event variability, RMS variability, and low-frequency spectral flux (specifically in the 0-50, 50-100, and 100-200 $\mathrm{Hz}$ sub-bands). These features were correlated with one another (Table 10 in Appendix B) and highly-correlated features were removed. This resulted in Flux 0-50, Flux 50-100, and RMS Variability being removed from the model.

Modelling ratings. Participants' ratings of wanting to move from both EEG 
and tapping blocks were averaged together and entered into a multiple linear regression with Beat Salience, Event Variability, and Flux 0-50 (Table 2). A marginally-significant regression equation was found $(F(3,26)=2.314, p=.099$, $\left.\mathrm{R}^{2}=.211\right)$. Beat Salience was a marginally-significant predictor, whereby Ratings of Wanting to Move increased with decreasing Beat Salience.

Table 2. Ratings model using acoustic features

\begin{tabular}{llll}
\hline Feature & $\beta$ & $t$ & $p$ \\
\hline Beat Salience & -0.3471 & -1.749 & $.092 \dagger$ \\
Event Variability & 0.3516 & 1.533 & .13 \\
Flux 100-200 Hz & 0.1491 & 0.7199 & .47 \\
\hline
\end{tabular}

Modelling entrainment. The same features were then entered into a multiple linear regression with PMC entrainment at the beat frequency as the dependent variable. A marginally-significant regression equation was found $(F(3,26)=$ 2.393, $\left.p=.091, \mathrm{R}^{2}=.216\right)$. Event Variability was a significant predictor, whereby entrainment decreased with increasing event variability (Figure 13).

Table 3. Ratings model applied to premotor entrainment to the beat.

\begin{tabular}{llll}
\hline Feature & $\beta$ & $t$ & $p$ \\
\hline Beat Salience & 0.01159 & 0.0586 & .95 \\
Event Variability & -0.5432 & -2.377 & $.025^{*}$ \\
Flux 100-200 Hz & 0.2487 & 1.205 & .23 \\
\hline
\end{tabular}

\section{Model of Tapping Variability}

Feature selection. For movement synchronization, previous work has found that pulse clarity, percussiveness, and low-frequency flux (especially in the 0$50,50-100$, and $100-200 \mathrm{~Hz}$ sub-bands) are related to regularity of movement 


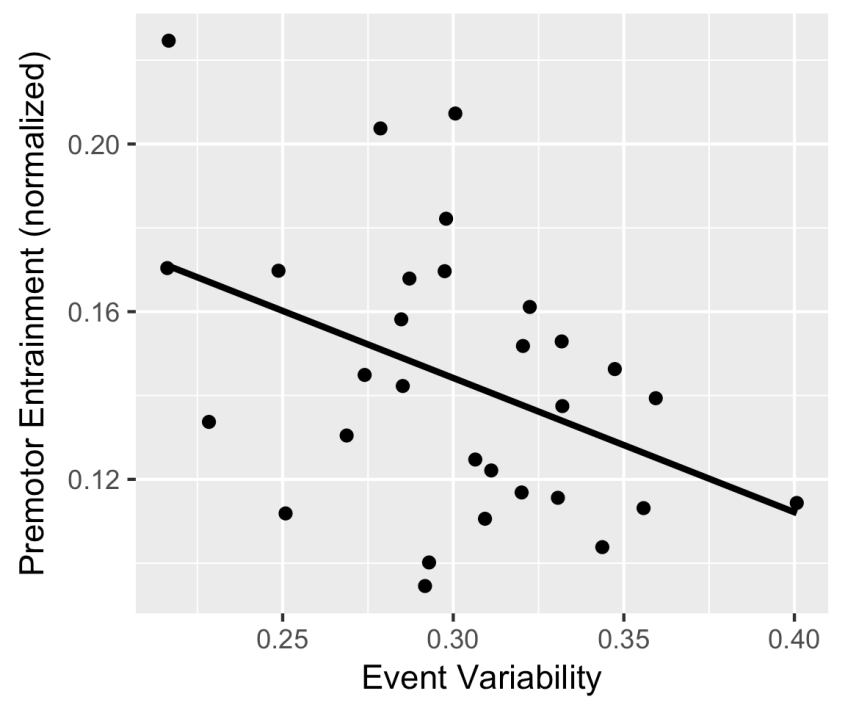

Figure 13. Event variability and PMC entrainment. PMC entrainment to the beat was negatively correlated with event variability $(r=-.41, p=.023)$.

(Burger et al., 2012; Stupacher et al., 2016). These features were correlated with one another (Table 11 in Appendix B) and highly-correlated features were removed. This resulted in Flux 0-50 and Flux 50-100 being removed from the model.

Modelling Tapping. Using these variables in a linear model to predict tapping variability, a significant regression equation was not found $(F(3,26)=2.259, p$ $\left.=.10, \mathrm{R}^{2}=.207\right)$. Percussiveness was a significant predictor, whereby tapping variability increased (i.e., performance got worse) with increasing Percussiveness. Indeed, Percussiveness was significantly correlated with Tapping Variability $(r$ $=-.39, p=.035)$.

Table 4. Tapping variability model using acoustic features

\begin{tabular}{llll}
\hline Feature & $\beta$ & $t$ & $p$ \\
\hline Pulse Clarity & 0.2364 & 1.293 & .20 \\
Percussiveness & -0.3729 & -2.131 & $.042^{*}$ \\
Flux 0-50 Hz & 0.1479 & 0.81 & .42 \\
\hline
\end{tabular}


Modelling entrainment. This model was then applied to PMC entrainment at the beat frequency. The regression equation was not significant $(F(3,26)=$ $\left.1.462, p=.24, \mathrm{R}^{2}=.144\right)$. Percussiveness was a marginally-significant predictor, whereby PMC entrainment increased with increasing Percussiveness.

Table 5. Tapping model applied to PMC entrainment to the beat.

\begin{tabular}{llll}
\hline Feature & $\beta$ & $t$ & $p$ \\
\hline Pulse Clarity & 0.1082 & 0.5698 & .57 \\
Percussiveness & 0.3597 & 1.979 & $.058 \dagger$ \\
Flux 0-50 Hz & -0.05386 & -0.2839 & .77 \\
\hline
\end{tabular}

\section{Model of PMC Entrainment}

Feature selection. As an exploratory analysis to see what features might be related to neural entrainment, all the previously-mentioned features were entered into a correlation table (Table 12 in Appendix B). The features that were most-correlated with PMC Entrainment were Event Variability $(r=-.41$, $p=.023)$ and Percussiveness $(r=.36, p=.054)$. These two features were also not correlated with one another $(r=.055, p=.77)$.

Modelling entrainment. Event Variability and Percussiveness were then entered into a fixed regression model with PMC Entrainment as the dependent variable. The model was found to be significant $\left(F(2,27)=6.197, p=.0060, \mathrm{R}^{2}\right.$ $=.315$ ), whereby PMC Entrainment increased with decreasing Event Variability and increasing Percussiveness.

Table 6. Premotor entrainment model using acoustic features

\begin{tabular}{llll}
\hline Feature & $\beta$ & $t$ & $p$ \\
\hline Event Variability & -0.4346 & -2.723 & $.011^{*}$ \\
Percussiveness & 0.3796 & 2.379 & $.024^{*}$ \\
\hline
\end{tabular}




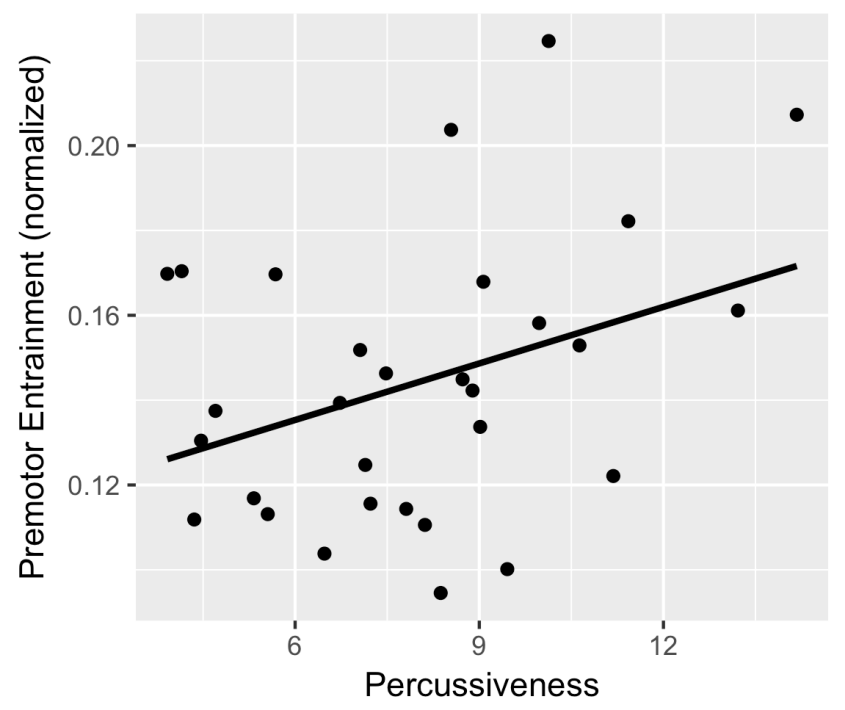

Figure 14. Percussiveness and PMC entrainment. PMC entrainment to the beat was marginally correlated with Percussiveness $(r=.36, p=.054)$.

\section{Discussion}

The current study found that some acoustic features previously associated with feelings of groove and movement synchronization are also related to neural entrainment in PMC. Specifically, event variability, which characterizes the variability of note onsets, was negatively correlated with PMC entrainment, and percussiveness, characterized by a steeper slope of the amplitude envelope for note onsets, was positively correlated with PMC entrainment. A signal that has more variable note onsets likely means that the rhythm is more complex and unpredictable. This would make it more difficult to entrain to the beat frequency. By contrast, the steeper attack slope that characterizes high percussiveness would support the predictability of a rhythm, since note onsets would be marked more precisely in time.

These observations may be understood from the perspective of predictive coding models as applied to feelings of groove (Vuust \& Witek, 2014). This theory posits that the brain draws on existing internal models in an attempt to describe the auditory input and predict upcoming beats. When the internal model is an 
imperfect match for the input, the resulting prediction error is used to update the model. Input that is too simple or too complex would not be associated with any prediction error, because the internal model is either a good match or is nonexistent. In terms of neural activity while attempting to model the metric structure of a complex rhythm, entrainment is expected to decrease as the system tries to reconcile its model with the input.

If higher levels of event variability increase the complexity of a rhythm, then the current pattern of results would be expected. However, if event variability became too high, stimuli should theoretically fail to activate any existing models, leading to an inverted- $\mathrm{U}$ relationship. A similar inverted-U relationship has been documented between syncopation and PMC entrainment (Witek et al., 2014). It seems unlikely that any of the stimuli in the current study were complex enough to reveal the other half of this theoretical function.

The finding that beat salience seemed to be negatively related to ratings of wanting to move was unexpected, as this is the opposite relationship that has been found in previous work (Madison et al., 2011). One possible explanation is that beat salience displays an inverted-U relationship with groove, and these stimuli only represent moderate and high beat salience, while other studies using natural music represented low and moderate beat salience. The stimuli used here were drumbeats that were homogeneous, and the beat was very easy to perceive in all excerpts. In contrast, Madison et al. (2011) used natural music in many different rhythmic styles (e.g., jazz, Indian, West African). Excerpts low in beat salience were likely rhythmically complex, making it difficult to perceive a beat.

\section{Conclusions}

The current study finds that beat salience is related to the desire to move, albeit in the opposite direction from previous work (Madison et al., 2011). However, given the differences in stimuli between the two studies, it is likely that each study represents a different half of an inverted-U relationship between beat 
salience and groove. The current study also replicates previous work on movement synchronization where percussiveness is related to less variability when moving along to the beat of music (Burger et al., 2012, 2013). Greater PMC entrainment was elicited for drum patterns that had low event variability and high percussiveness, suggesting that acoustic features related to groove and movement are also related to entrainment. 


\section{General Discussion}

This dissertation investigated aspects of music that drive the entrainment of neurons in motor areas of the brain. In three studies, it described some of the psychological, musical, and acoustic aspects of music that contribute to PMC entrainment. In Study 1, songs that elicited greater feelings of groove also elicited greater entrainment in PMC. In Study 2, rhythms with moderate degrees of syncopation seemed to elicit less entrainment at beat-related frequencies in PMC and auditory cortex. In Study 3, drum patterns with greater variability in their onset signals were predictive of less entrainment in PMC, whereas steeper onset slopes were predictive of more. Taken together, these studies offer a unique perspective on the neural dynamics of listening to music, and offer new insights regarding how music can drive the desire to move.

Music provides a formidable temporal framework with which to synchronize movement. Simply listening to music has been found to elicit PMC activation (Chen et al., 2008), suggesting a neural substrate for movement synchronization. Rhythms with regular temporal structure have been found to facilitate synchronization as well as PMC activation (Grahn \& Brett, 2007). Music that facilitates movement synchronization has also been associated with groove or the desire to move (Janata et al., 2012). Thus, it was hypothesized that greater populations of neurons in PMC would synchronize their firing under conditions of high-groove.

It is important to note, however, that while the increased BOLD response found in previous studies implies that more neurons are firing in a particular region, fMRI cannot provide any indication regarding whether they are firing synchronously. More neurons may be firing in PMC during beat perception, but whether they are entrained cannot be ascertained. Source localization using tools with superior temporal resolution such as EEG provide a unique opportunity to study simultaneously the location and temporal dynamics of neural processing. While the spatial resolution of 128-channel EEG is far inferior to that of fMRI, existing fMRI work can guide our expectations about where ac- 
tivity should be localized during certain tasks.

\section{Beat Complexity}

While Study 1 of this dissertation found more neural entrainment while listening to high-groove music, Studies 2 and 3 found less entrainment to rhythms under conditions that have been associated with high-groove such as syncopation and event variability. Here I offer a unifying framework for interpreting this pattern of findings that involves a consideration of stimulus complexity across the studies. Based on predictive coding theories (Vuust \& Witek, 2014), rhythms that are too low or too high in complexity do not elicit any prediction error, because the correspondence between the model and the input is either excellent or nonexistent. Rhythms of medium complexity elicit some prediction error because the system can only partially model the input.

There are many ways to measure the complexity of a rhythm or musical stimulus, some of which have been investigated in this dissertation such as syncopation, event variability, and percussiveness. Premotor cortices are active when listening to beat rhythms (Chen et al., 2008; Grahn \& Brett, 2007), and presumably the active motor programs are those which will aid in predicting the auditory input (Patel \& Iversen, 2014). How the complexity of the stimulus affects the entrainment of these motor programs likely has some influence on feelings of groove. I consider beat complexity to be the extent to which a rhythm creates tension with these pre-existing motor programs. That is, beat complexity increases with rhythm complexity as long as the listener is able to maintain a beat (i.e., as long as there is a compatible motor program). For higher rhythm complexity, beat complexity drops off because a beat is no longer being perceived. Table 7 summarizes the various stimuli in this dissertation in terms of beat complexity. Categorized in this way, all stimuli map well onto the inverted-U relationship with groove that has been previously-reported (Sioros et al., 2014; Witek et al., 2014), and unifies the pattern of results seen across the three studies. In the section that follows, results will be reconsidered from the perspective 
of beat complexity.

Table \%. Beat complexity

\begin{tabular}{|c|c|c|c|}
\hline Beat Complexity & low & high & low \\
\hline $\begin{array}{l}\text { Rhythmic } \\
\text { Complexity }\end{array}$ & low & medium & high \\
\hline $\begin{array}{l}\text { Feelings of } \\
\text { Groove }\end{array}$ & low & high & low \\
\hline $\begin{array}{l}\text { Movement } \\
\text { Synchronization }\end{array}$ & high & medium & low \\
\hline $\begin{array}{l}\text { Premotor } \\
\text { Entrainment }\end{array}$ & high & medium & low \\
\hline Studies' Stimuli & & $\begin{array}{l}1 \text { (mid groove) } \\
1 \text { (high groove) }\end{array}$ & 1 (low groove) \\
\hline & $\begin{array}{l}2 \text { (low sync.) } \\
3 \text { (low event } \\
\text { var.) }\end{array}$ & $\begin{array}{l}2 \text { (mid sync.) } \\
3 \text { (high event var.) }\end{array}$ & 2 (high sync.) $)^{19}$ \\
\hline
\end{tabular}

In Study 1, mid- and high-groove excerpts tended to have more salient temporal structure, and low-groove excerpts usually did not have drums, which made it more difficult to find the beat. Low-groove excerpts from this collection of stimuli are also more difficult to synchronize with when tapping along (Janata et al., 2012). In terms of being able to model the metric structure, it was likely too complex or undefined to elicit the activation of an existing model. Thus, entrainment to the beat was lower for these low-groove excerpts.

In Study 2, neural entrainment to the beat seemed to be highest for low degrees of syncopation and lowest for moderate degrees. This is somewhat perplexing in that moderate levels of syncopation have previously been associated with

\footnotetext{
${ }^{19}$ For the highly-syncopated rhythms in Study 2, neural entrainment seemed to be greater than this table would suggest. This was likely due to the rhythmic hi-hat that was part of these stimuli.
} 
the highest ratings of wanting to move (Sioros et al., 2014; Witek et al., 2014) and also the greatest prediction error (Vuust \& Witek, 2014). The reduced entrainment found with moderate syncopation may have arisen due to the relative complexity involved in finding the beat. For high degrees of syncopation, we would expect higher beat complexity and less neural entrainment, but this did not seem to be the case in Study 2. One possible explanation is that listeners chose to focus their attention on the hi-hat, which clearly outlined the beat in all conditions. In this case, highly-syncopated stimuli would in fact be relatively low in beat complexity. By comparison, focusing on the hi-hat while discarding other aspects of the rhythm may have been more challenging in the moderate syncopation condition because the two rhythms were more compatible with one another, but not as compatible as for low syncopation.

In Study 3, PMC entrainment to the beat was lowest for high levels of event variability in the drum patterns. Higher event variability can be thought of as higher complexity, meaning that the brain's ability to model the stimulus becomes reduced. The stimuli here were all drum beats with very salient metrical structure, and there were not any rhythms that deliberately pushed against the intended meter (as was the case for moderately-syncopated stimuli in Study 2). As a result, it seems likely that the range of event variability in these stimuli consisted only of low- and medium-complexity categories. If this were the case, it would be predicted that drum patterns with even greater event variability would elicit lower ratings of groove.

The tapping results from Studies 2 and 3 can also be interpreted with regard to complexity, where participants are more variable when tapping along to higher complexity rhythms. Predicting the temporal location of upcoming beats and note onsets in a complex rhythm is difficult, so it makes sense that the ability to tap along in time be worse. If the beat is more obvious, then it is easier to synchronize movement. This was the case for Studies 2 and 3, and has been found previously for the stimuli in Study 1 (Janata et al., 2012). Together with the entrainment findings, it seems that high complexity rhythms give rise to less PMC entrainment and also less regular motor output. This fits with previous 
fMRI work finding premotor BOLD response to rhythms that participants were better at tapping along to (Grahn \& Brett, 2007).

\section{Endogenous Oscillations}

There are rich connections between PMC and the BG that facilitate the coordination and execution of action. So, some of the PMC effects here may have their origins there. The BG have been found to be active during both the perception and production of musical rhythms (Kung, Chen, Zatorre, \& Penhune, 2013). Greater BG activity (particularly in the putamen ${ }^{20}$ ) have been specifically associated with perceiving rhythms which have a beat (Grahn \& Brett, 2007), suggesting a role of these structures in synchronizing movement with music. Rhythms which elicited greater BG activity also afforded superior tapping performance (Grahn \& Brett, 2007). When the BG are compromised, as in Parkinson's disease, it has been proposed that the corresponding disruption of the basal ganglia-thalamo-cortical motor circuit (DeLong, 1990) causes a decreased ability for voluntary movement. This suggests that the BG may be involved in the endogenous generation of oscillatory activity used to coordinate music and movement. As part of this circuit, the putamen receives projections from PMC, and has direct projections onto brainstem nuclei involved in motor output $^{21}$. Motor plans activated in PMC can effect movement via this circuit, but overt movement does not seem to be necessary for BG activity (Kung et al., 2013).

Using synchronization-continuation tasks ${ }^{22}$, increased activity in the putamen and SMA has been found during the continuation phase of these tasks (Lewis, Wing, Pope, Praamstra, \& Miall, 2004), suggesting that these structures are implicated in the generation of endogenous oscillations. Beta-band activity has

\footnotetext{
${ }^{20}$ The putamen is a structure in the dorsal striatum of the BG.

${ }^{21}$ The putamen has direct projections onto the internal segment of the globus pallidus and the substantia nigra pars reticulata, which are both involved in motor output.

${ }^{22}$ In a synchronization-continuation task, the participant synchronizes movements (usually tapping) with a stimulus (e.g., a metronome), and continue the movement after the stimulus has stopped.
} 
been suggested to facilitate oscillatory communication between brain regions (Merchant et al., 2015), and both human and animal work have suggested a role for the beta-band in the endogenous generation of temporal prediction in isochronous sequences (Bartolo, Prado, \& Merchant, 2014; Fujioka et al., 2012). Local field potential (LFP) recordings in monkeys have revealed that different neural populations in the putamen code for different durations of time intervals (Bartolo et al., 2014).

It is important to remember that groove is likely a difficult thing to experience to its full potential without actually moving when you feel the desire to move. The experiments presented here all asked participants to sit still, so that movement artifacts would not disrupt the EEG recording. This means that these studies may have been reducing the amount of groove felt by participants. Indeed, increased activity in PMC is seen during tapping tasks (Chen et al., 2008; Nozaradan et al., 2015). It has been suggested that syncopation, where metrically-important beats are left empty, invites the listener to move on these empty beats as a way of "filling in" the rest of the meter (Witek, 2017). This "embodiment" of the rhythm (Burger et al., 2013) may increase feelings of groove beyond passive listening.

\section{The Groove Pathway}

Taken together with previous work, I propose a cortical "groove pathway", consisting of a set of brain areas, that when activated will elicit the desire to move along with music. First, sound enters the ear and is converted into a pattern of neural firing based on the movement of the basilar membrane. Neurons in auditory cortex begin to synchronize with this pattern of firing. Organized as a bank of frequency-tuned oscillators, populations of neurons begin to entrain to the oscillatory structure of the sound input. Of particular interest here are lower frequency oscillations corresponding to the amplitude envelope at the beat level and related frequencies. The complexity of the input does not matter here; auditory areas should entrain well to any kind of input, regardless of its 
structure.

Depending on the structure of the auditory firing pattern, connected brain areas with matching neural architectures may begin to synchronize their firing as well. The extent to which this occurs is dependent on the specific connectivity and firing patterns of the neurons there, so they will only resonate if the auditory firing pattern is sufficiently similar. In the case of PMC, its connectivity and firing patterns are presumably tuned for body movements - such as walking and talking - that all have a particular timing structure. For example, walking involves timing the neural firing of motor neurons to activate the muscles of two legs as they take turns stepping. The higher-order structure of this pattern of firing is similar to music, where, for example, the repetition of two notes would lead to a duple pattern. Synchronization of auditory and motor neural populations has been suggested to be done via beta- and gamma-band oscillations (Merchant et al., 2015), which have been previously implicated in beat perception (Fujioka et al., 2012; Snyder \& Large, 2005).

The extent to which the premotor model is a good match with input will determine the extent of entrainment in PMC. A good match would result in high entrainment to the beat, whereas no match would not elicit any. A partial match could still drive the entrainment of a motor model, but would involve some prediction error between them, and an attempt would be made to reconcile this. Vuust and Witek (2014) propose that one way to do this is to actually move the body in accordance with the model in an attempt to minimize prediction error. In a sense, the brain is attempting to increase entrainment of its model by involving primary motor and proprioceptive neurons. This drive to minimize prediction error by emphasizing the beat with the body is the feeling of groove. While it is beyond the scope of this dissertation, the positive feeling that people experience when they "groove" to the music may arise as a function of minimizing prediction error. If we think of the brain as a prediction machine (Friston, 2010), then grooving to the music can be thought of as the brain leveraging its various modalities in order to achieve its mission to model the world around it. The brain's reward systems have been suggested to play a role here 
(Vuust \& Witek, 2014).

The degree of mismatch between the model and the input has an inverted-U relationship with the desire to emphasize the model. When the model almost fits the input, the reaction is to emphasize the model in order to make it fit. This model could reside anywhere in the brain and involve any combination of systems, depending on the input. Phenomenologically, the activation of these systems is experienced as a desire to carry out those programs. In groove, this model resides with systems that control movement of the body, which means that emphasizing the model entails neural firing that enacts movement.

\section{Limitations and Future Directions}

In studies 2 and 3, trials were not repeated (at least not at the same tempo), which precluded an evoked analysis where trials were first averaged together in the time-domain before assessing entrainment. Averaging together in the time domain restricts the analysis to phase-locked activity (Snyder \& Large, 2005), and this is the method used in other work assessing steady-state evoked potentials (SSEPs) in response to rhythmic stimuli (Nozaradan et al., 2011). Because tempo was controlled, repeating trials at the same tempo would not have been feasible as experimental sessions would have run far too long. A future study could drop the tempo control and assess SSEPs instead of localized entrainment. Doing so would provide a valuable complement to the current work.

An important limitation of using ICA with EEG is that sources of activity below the surface of the cortex (e.g., BG activity) are not well-resolved. This means that ICA will not often return a reliable IC that is localized to the BG. This makes it difficult to probe the entire proposed groove network using EEG. fMRI methods are not susceptible to this limitation, but as previously discussed they do not have the temporal resolution to be able to assess entrainment. However, whole-brain computational modelling using fMRI allows one to study the effective connectivity of different regions during a task, making it possible 
to investigate how seemingly disparate brain regions might operate in tandem. Combining data and results from all of these methods will likely be necessary to elucidate this network further. Studies involving EEG and fMRI recordings of the same participants and stimuli would be particularly informative.

In Parkinson's Disease (PD), cell death in the BG makes voluntary movement difficult, but direct auditory-motor links provide an alternative way of driving the motor system. Patients who have difficulty walking are able to walk with improved pace and stride length when they walk along to the beat of the music (Thaut \& Abiru, 2010). The extent to which auditory areas are able to drive the motor system likely depends on many factors to do with the choice of music. Systems exist for aiding music selection based on the optimal tempo for a particular person, the strength of the beat, and individual preferences (Li et al., 2010). Investigating these and other aspects of music with respect to their ability to drive PMC entrainment could offer insight into this treatment and help to optimize it. Additionally, since there is cell death in the BG in PD, it provides a unique opportunity to further elucidate the role of the BG in the perception of groove. The BG seem necessary for beat perception (Merchant et al., 2015), but whether they or other brain regions are necessary to feel groove remains an open question.

Music provides an excellent temporal framework for synchronization, and also a unique opportunity to study the temporal dynamics of neural processing. It is becoming apparent that endogenous oscillatory activity in the brain is an important mechanism for communication between brain regions (Buzsaki, 2006). How might the results presented here generalize to other domains? Speaking is another very rhythmic activity that humans engage in, and there are great public speakers who can command a crowd with the timbre and timing of their voice, in much the same way as a great singer. Neural oscillations have been proposed to be involved in speech processing (Giraud \& Poeppel, 2012; but see Obleser, Herrmann, \& Henry, 2012). Moreover, like music, speech has a temporal structure that lends well to the firing patterns of existing neural architecture, and provides an opportunity for a stimulus to be a partial match for an existing 
model. Just like syncopation creates groove, it seems likely that the phrasing and timing of a speech could focus a listener's attention to specific points in time, giving the speaker an opportunity to emphasize or de-emphasize certain words depending on the intended message or effect. By leveraging the expertise of the motor system, a listener may be more attuned to these timing patterns and be better able to understand what is being said.

\section{Conclusion}

The current dissertation investigated how aspects of music that elicit feelings of groove can drive neural entrainment. It was found that groove is related the extent of entrainment to the beat in premotor areas of the brain. Results across three studies suggest that neural entrainment is related to the rhythmic complexity of the musical stimulus, where higher degrees of complexity result in less entrainment. However, while groove has been found to relate to acoustic and rhythmic complexity, I propose "beat complexity" as way of describing the pattern of behavioural and neural results in research on groove. At optimal levels of beat complexity, groove ratings are highest and premotor entrainment to the beat is reduced, as the brain attempts to reconcile the differences between rhythm and meter. I suggest that the compulsion to move is a natural consequence of this process, whereby the system attempts to emphasize the model by moving along with it. These results add to the existing literature on rhythm perception and neural entrainment by offering a mechanism by which feelings of groove can originate. 
Appendix A

Table 8. List of stimuli (Study 1)

\begin{tabular}{|c|c|c|c|c|}
\hline Song & Artist & $\begin{array}{l}\text { Groove } \\
\text { Rating }\end{array}$ & $\begin{array}{l}\text { Groove } \mathrm{T} \\
\text { Category }\end{array}$ & $\begin{array}{r}\text { Tempo } \\
(\mathrm{Hz})\end{array}$ \\
\hline Superstition & Stevie Wonder & 108.7 & high & 1.667 \\
\hline It's A Wrap (Bye, Bye) & FH1 (Funky Hobo \#1) & 105.9 & high & 1.533 \\
\hline Flash Light & Parliament & 105.1 & high & 1.767 \\
\hline Lady Marmalade & LaBelle & 102.5 & high & 1.917 \\
\hline Up for the Downstroke & $\begin{array}{l}\text { The Clinton } \\
\text { Administration }\end{array}$ & 102.4 & high & 1.583 \\
\hline $\begin{array}{l}\text { Mama Cita } \\
\text { (Instrumental) }\end{array}$ & Funk Squad & 101.6 & high & 1.583 \\
\hline Music & Leela James & 101.1 & high & 1.633 \\
\hline If I Ain't Got You & Alicia Keys & 98.7 & high & 2 \\
\hline Sing, Sing, Sing & $\begin{array}{l}\text { Benny Goodman and His } \\
\text { Orchestra }\end{array}$ & 97.4 & high & 1.8 \\
\hline In the Mood & $\begin{array}{l}\text { Glenn Miller and His } \\
\text { Orchestra }\end{array}$ & 96.9 & high & 2.733 \\
\hline Summertime & $\begin{array}{l}\text { Ella Fitzgerald and Louis } \\
\text { Armstrong }\end{array}$ & 67.9 & mid & \\
\hline $\begin{array}{l}\text { What a Wonderful } \\
\text { World }\end{array}$ & Louis Armstrong & 66.4 & mid & 1.233 \\
\hline $\begin{array}{l}\text { How High the Moon } \\
\text { (1st take) }\end{array}$ & Ella Fitzgerald & 65.2 & mid & 1.883 \\
\hline Squeeze & $\begin{array}{l}\text { Robert Randolph and the } \\
\text { Family Band }\end{array}$ & 63.4 & mid & 1.95 \\
\hline The Child Is Gone & Fiona Apple & 62.3 & mid & 1.083 \\
\hline Run / Immortal & Beth Hart & 60.8 & mid & 1.917 \\
\hline Freedom of the Road & Martin Sexton & 59.7 & mid & 2.45 \\
\hline Can't Let Go & Lucinda Williams & 58.9 & mid & 1.583 \\
\hline
\end{tabular}




\begin{tabular}{|c|c|c|c|c|}
\hline Song & Artist & $\begin{array}{l}\text { Groove } \\
\text { Rating }\end{array}$ & $\begin{array}{l}\text { Groove T } \\
\text { Category }\end{array}$ & $\begin{array}{r}\text { Tempo } \\
(\mathrm{Hz})\end{array}$ \\
\hline Down With Love & Blossom Dearie & 57 & mid & 2.333 \\
\hline The Girl From & Astrud Gilberto, Joao & 57 & mid & 2.15 \\
\hline Ipanema & Gilberto, and Stan Getz & & & \\
\hline Space Oddity & David Bowie & 38.7 & low & 2.267 \\
\hline Ray Dawn Balloon & Trey Anastasio & 38.5 & low & 2.767 \\
\hline Druid Fluid & $\begin{array}{l}\text { Yo-Yo Ma, Mark } \\
\text { O'Connor, and Edgar } \\
\text { Meyer }\end{array}$ & 38.1 & low & 1.95 \\
\hline Flandyke Shore & The Albion Band & 36.5 & low & 1.5 \\
\hline Citi Na GCumman & $\begin{array}{l}\text { William Coulter and } \\
\text { Friends }\end{array}$ & 35.2 & low & \\
\hline Dawn Star & Dean Magraw & 34.8 & low & \\
\hline Fortuna & Kaki King & 32.6 & low & \\
\hline Beauty of the Sea & The Gabe Dixon Band & 32.1 & low & 1.9 \\
\hline Sweet Thing & Alison Brown & 30.9 & low & \\
\hline Hymn for Jaco & Adrian Legg & 29.3 & low & \\
\hline
\end{tabular}

Note. Excerpts were selected based on the groove ratings from Janata et al. (2012). Excerpts without a marked tempo were removed during analysis due to having a tempo that was not consistent throughout the excerpt.

Table 9. List of included data (Study 1)

\begin{tabular}{llll}
\hline Participant & Premotor Cortex & Auditory Cortex & Visual Cortex \\
\hline 1 & N/A & $2.09 \mathrm{E}-12$ & $7.57 \mathrm{E}-11$ \\
2 & N/A & N/A & $8.24 \mathrm{E}-14$ \\
3 & $3.53 \mathrm{E}-18$ & $9.04 \mathrm{E}-16$ & $2.15 \mathrm{E}-12$ \\
4 & $1.51 \mathrm{E}-15$ & $2.05 \mathrm{E}-14$ & $2.73 \mathrm{E}-13$ \\
5 & $9.07 \mathrm{E}-15$ & $1.43 \mathrm{E}-12$ & $2.46 \mathrm{E}-11$ \\
6 & $1.96 \mathrm{E}-09$ & $6.85 \mathrm{E}-10$ & $1.55 \mathrm{E}-14$
\end{tabular}




\begin{tabular}{llll}
\hline Participant & Premotor Cortex & Auditory Cortex & Visual Cortex \\
\hline 7 & $4.98 \mathrm{E}-18$ & N/A & $9.30 \mathrm{E}-16$ \\
8 & $5.31 \mathrm{E}-13$ & N/A & $1.47 \mathrm{E}-14$ \\
9 & $2.94 \mathrm{E}-14$ & $2.78 \mathrm{E}-15$ & $1.08 \mathrm{E}-17$ \\
10 & $5.66 \mathrm{E}-17$ & N/A & $4.47 \mathrm{E}-17$ \\
11 & $1.40 \mathrm{E}-13$ & N/A & $3.34 \mathrm{E}-11$ \\
\hline
\end{tabular}

Note. This table lists $p$-values for $t$-tests of whether spectral peaks were significantly above the noise floor for each participant and brain region. A value of $\mathrm{N} / \mathrm{A}$ indicates that no component was localized to the given region, thus an entrainment value of 0 was assigned. There were no cases of a component that was localized to a region but did not have its spectral peaks of interest significantly above the noise floor. 


\section{Appendix B}

In all tables, asterisks represent p-values less than .001 (***), .01 (**), .05 (*), and .1 $(\dagger)$.

Table 10. Correlations of acoustic features for the ratings model.

\begin{tabular}{|c|c|c|c|c|c|c|}
\hline & $\begin{array}{l}\text { Wanting } \\
\text { to Move }\end{array}$ & $\begin{array}{l}\text { Beat } \\
\text { Salience }\end{array}$ & $\begin{array}{l}\text { Event } \\
\text { Varia- } \\
\text { bility }\end{array}$ & $\begin{array}{l}\text { RMS } \\
\text { Varia- } \\
\text { bility }\end{array}$ & $\begin{array}{l}\text { Flux } \\
0-50 \\
\mathrm{~Hz}\end{array}$ & $\begin{array}{l}\text { Flux } \\
50-100 \\
\mathrm{~Hz}\end{array}$ \\
\hline $\begin{array}{l}\text { Wanting } \\
\text { to Move }\end{array}$ & & & & & & \\
\hline $\begin{array}{l}\text { Beat } \\
\text { Salience }\end{array}$ & -0.19 & & & & & \\
\hline $\begin{array}{l}\text { Event } \\
\text { Varia- } \\
\text { bility }\end{array}$ & 0.28 & $0.43^{*}$ & & & & \\
\hline $\begin{array}{l}\text { RMS } \\
\text { Varia- } \\
\text { bility }\end{array}$ & 0.25 & -0.01 & $0.49^{* *}$ & & & \\
\hline $\begin{array}{l}\text { Flux 0-50 } \\
\mathrm{Hz}\end{array}$ & 0.21 & 0.00 & 0.27 & $0.74^{* * *}$ & & \\
\hline $\begin{array}{l}\text { Flux } \\
50-100 \mathrm{~Hz}\end{array}$ & 0.24 & 0.01 & $0.31 \dagger$ & $0.76^{* * *}$ & $1.00^{* * *}$ & \\
\hline $\begin{array}{l}\text { Flux } \\
100-200 \mathrm{~Hz}\end{array}$ & $0.31 \dagger$ & 0.03 & $0.50^{* *}$ & $0.83^{* * *}$ & $0.86^{* * *}$ & $0.88^{* * *}$ \\
\hline
\end{tabular}


Table 11. Correlations of acoustic features for the tapping model

\begin{tabular}{|c|c|c|c|c|c|}
\hline & $\begin{array}{l}\text { Tapping } \\
\text { Varia-bility }\end{array}$ & $\begin{array}{l}\text { Pulse } \\
\text { Clarity }\end{array}$ & $\begin{array}{l}\text { Percuss- } \\
\text { iveness }\end{array}$ & $\begin{array}{l}\text { Flux } \\
0-50 \mathrm{~Hz}\end{array}$ & $\begin{array}{l}\text { Flux } \\
50-100 \\
\mathrm{~Hz}\end{array}$ \\
\hline \multicolumn{6}{|l|}{ Tapping } \\
\hline \multicolumn{6}{|l|}{ Variab-ility } \\
\hline Pulse Clarity & 0.21 & & & & \\
\hline $\begin{array}{l}\text { Percussi- } \\
\text { veness }\end{array}$ & $-0.39^{*}$ & -0.05 & & & \\
\hline Flux $0-50 \mathrm{~Hz}$ & 0.09 & -0.29 & -0.02 & & \\
\hline Flux 50-100 & 0.06 & -0.29 & 0.03 & $1.00^{* * *}$ & \\
\hline \multicolumn{6}{|l|}{$\mathrm{Hz}$} \\
\hline $\begin{array}{l}\text { Flux 100-200 } \\
\text { Hz }\end{array}$ & 0.00 & -0.18 & 0.24 & $0.86^{* * *}$ & $0.88^{* * *}$ \\
\hline
\end{tabular}

Table 12. Correlation table of acoustic features and premotor entrainment to the beat.

\begin{tabular}{|c|c|c|c|c|c|c|}
\hline & $\begin{array}{l}\text { PMC } \\
\text { Entrain- } \\
\text { ment }\end{array}$ & $\begin{array}{l}\text { Beat Pulse } \\
\text { SalienceClarity }\end{array}$ & $\begin{array}{l}\text { Event } \\
\text { Varia- } \\
\text { bility }\end{array}$ & $\begin{array}{l}\text { RMS } \\
\text { Percuss- Varia- } \\
\text { iveness bility }\end{array}$ & $\begin{array}{l}\text { Flux } \\
0-50 \\
\mathrm{~Hz}\end{array}$ & $\begin{array}{l}\text { Flux } \\
50- \\
100 \\
\mathrm{~Hz}\end{array}$ \\
\hline $\mathrm{PMC}$ & & & & & & \\
\hline $\begin{array}{l}\text { Entrain- } \\
\text { ment }\end{array}$ & & & & & & \\
\hline $\begin{array}{l}\text { Beat } \\
\text { Salience }\end{array}$ & -0.21 & & & & & \\
\hline $\begin{array}{l}\text { Pulse } \\
\text { Clarity }\end{array}$ & 0.11 & 0.14 & & & & \\
\hline
\end{tabular}




\begin{tabular}{|c|c|c|c|c|c|c|c|c|}
\hline & $\begin{array}{l}\text { PMC } \\
\text { Entrain- } \\
\text { ment }\end{array}$ & $\begin{array}{l}\text { Beat } \\
\text { Salience }\end{array}$ & $\begin{array}{c}\text { Pulse } \\
\text { eClarity }\end{array}$ & $\begin{array}{l}\text { Event } \\
\text { Varia- } \\
\text { bility }\end{array}$ & $\begin{array}{l}\text { Percuss- } \\
\text { iveness }\end{array}$ & $\begin{array}{l}\text { RMS } \\
\text { Varia- } \\
\text { bility }\end{array}$ & $\begin{array}{l}\text { Flux } \\
0-50 \\
\mathrm{~Hz}\end{array}$ & $\begin{array}{l}\text { Flux } \\
50- \\
100 \\
\mathrm{~Hz}\end{array}$ \\
\hline $\begin{array}{l}\text { Event } \\
\text { Varia- } \\
\text { bility }\end{array}$ & $-0.41^{*}$ & $0.43^{*}$ & 0.22 & & & & & \\
\hline $\begin{array}{l}\text { Percuss- } \\
\text { iveness }\end{array}$ & $0.36 \dagger$ & -0.09 & -0.05 & 0.06 & & & & \\
\hline $\begin{array}{l}\text { RMS } \\
\text { Varia- } \\
\text { bility }\end{array}$ & 0.02 & -0.01 & -0.02 & $0.49^{* *}$ & 0.27 & & & \\
\hline $\begin{array}{l}\text { Flux } \\
0-50 \mathrm{~Hz}\end{array}$ & -0.09 & 0.00 & -0.29 & 0.27 & -0.02 & $0.74^{* * *}$ & & \\
\hline $\begin{array}{l}\text { Flux } \\
50-100 \\
\mathrm{~Hz}\end{array}$ & -0.10 & 0.01 & -0.29 & $0.31 \dagger$ & 0.03 & $0.76^{* * *}$ & $1.00^{* * *}$ & \\
\hline $\begin{array}{l}\text { Flux } \\
100-200\end{array}$ & -0.02 & 0.03 & -0.18 & $0.50^{* *}$ & 0.24 & $0.83^{* * *}$ & $0.86^{* * *}$ & $0.88 * * *$ \\
\hline $\mathrm{Hz}$ & & & & & & & & \\
\hline
\end{tabular}




\section{References}

Arnal, L. H., Doelling, K. B., \& Poeppel, D. (2015). Delta-beta coupled oscillations underlie temporal prediction accuracy. Cerebral Cortex, 25(9), 3077-3085. https://doi.org/10.1093/cercor/bhu103

Bartolo, R., Prado, L., \& Merchant, H. (2014). Information Processing in the Primate Basal Ganglia during Sensory-Guided and Internally Driven Rhythmic Tapping. Journal of Neuroscience, 34(11), 3910-3923. https://doi.org/10.1523/ JNEUROSCI.2679-13.2014

Bell, A. J., \& Sejnowski, T. J. (1995). An Information-Maximization Approach to Blind Separation and Blind Deconvolution. Neural Computation, 7(6), 1129 1159. https://doi.org/10.1162/neco.1995.7.6.1129

Burger, B., Thompson, M. R., Luck, G., Saarikallio, S., \& Toiviainen, P. (2012). Music Moves Us: Beat-Related Musical Features Influence Regularity of MusicInduced Movement. Proceedings of the 12th International Conference on Music Perception and Cognition, 183-187.

Burger, B., Thompson, M. R., Luck, G., Saarikallio, S., \& Toiviainen, P. (2013). Influences of Rhythm- and Timbre-Related Musical Features on Characteristics of Music-Induced Movement. Frontiers in Psychology, 4(April), 183. https: //doi.org/10.3389/fpsyg.2013.00183

Buzsaki, G. (2006). Rhythms of the Brain. New York, NY: Oxford University Press.

Chen, J. L., Penhune, V. B., \& Zatorre, R. J. (2008). Listening to Musical Rhythms Recruits Motor Regions of the Brain. Cerebral Cortex, 18(12), 28442854. https://doi.org/10.1093/cercor/bhn042

Cirelli, L. K., Spinelli, C., Nozaradan, S., \& Trainor, L. J. (2016). Measuring neural entrainment to beat and meter in infants: Effects of music background. Frontiers in Neuroscience, 10(MAY), 1-11. https://doi.org/10.3389/fnins.2016. 
00229

Davies, M., Madison, G., Silva, P., \& Gouyon, F. (2013). The Effect of Microtiming Deviations on the Perception of Groove in Short Rhythms. Music Perception: An Interdisciplinary Journal, 30(5), 497-510.

Debener, S., Thorne, J., Schneider, T. R., \& Viola, F. C. (2010). Using ICA for the Analysis of Multi-Channel EEG Data. In Simultaneous eeg and fMRI: Recording, analysis, and application. Oxford University Press.

DeLong, M. R. (1990). Primate models of movement disorders of basal ganglia origin. Trends in Neurosciences, 13(7), 281-285. https://doi.org/10.1016/ 0166-2236(90)90110-V

Delorme, A., \& Makeig, S. (2004). EEGLAB: an open source toolbox for analysis of single-trial EEG dynamics including independent component analysis. Journal of Neuroscience Methods, 134, 9-21.

Delorme, A., Palmer, J., Onton, J., Oostenveld, R., \& Makeig, S. (2012). Independent EEG Sources Are Dipolar. PLoS ONE, 7(2), 1-14.

Demanuele, C., James, C. J., \& Sonuga-Barke, E. J. S. (2007). Distinguishing low frequency oscillations within the $1 /$ f spectral behaviour of electromagnetic brain signals. Behavioral and Brain Functions, 3, 1-14. https://doi.org/10. 1186/1744-9081-3-62

Engel, A. K., \& Fries, P. (2010). Beta-band oscillations-signalling the status quo? Current Opinion in Neurobiology, 20(2), 156-165. https://doi.org/10. 1016/j.conb.2010.02.015

Escoffier, N., Herrmann, C. S., \& Schirmer, A. (2015). Auditory rhythms entrain visual processes in the human brain: Evidence from evoked oscillations and event-related potentials. NeuroImage, 111, 267-276. https://doi.org/10.1016/j. neuroimage.2015.02.024

Fitch, W. T. (2016). Dance, music, meter and groove: A forgotten partner- 
ship. Frontiers in Human Neuroscience, 10(March), 64. https://doi.org/10. 3389/fnhum.2016.00064

Friston, K. (2010). The free-energy principle: a unified brain theory? Nature Reviews. Neuroscience, 11(2), 127-138. https://doi.org/10.1038/nrn2787

Fujioka, T., Trainor, L. J., Large, E. W., \& Ross, B. (2012). Internalized Timing of Isochronous Sounds Is Represented in Neuromagnetic Beta Oscillations. Journal of Neuroscience, 32(5), 1791-1802. https://doi.org/10.1523/JNEUROSCI. 4107-11.2012

Fujioka, T., Zendel, B. R., \& Ross, B. (2010). Endogenous neuromagnetic activity for mental hierarchy of timing. The Journal of Neuroscience : The Official Journal of the Society for Neuroscience, 30(9), 3458-3466. https://doi. org/10.1523/JNEUROSCI.3086-09.2010

Giraud, A.-L., \& Poeppel, D. (2012). Cortical oscillations and speech processing: emerging computational principles and operations. Nature Neuroscience, 15(4), 511-517. https://doi.org/10.1038/nn.3063

Grahn, J. A., \& Brett, M. (2007). Rhythm and beat perception in motor areas of the brain. Journal of Cognitive Neuroscience, 19(5), 893-906. https: //doi.org/10.1162/jocn.2007.19.5.893

Grahn, J. A., \& Rowe, J. B. (2009). Feeling the Beat: Premotor and Striatal Interactions in Musicians and Nonmusicians during Beat Perception. Journal of Neuroscience, 29(23), 7540-7548. https://doi.org/10.1523/JNEUROSCI. 2018-08.2009

Honing, H., Ladinig, O., Háden, G. P., \& Winkler, I. (2009). Is beat induction innate or learned: Probing emergent meter perception in adults and newborns using event-related brain potentials. Annals of the New York Academy of Sciences, 1169(Illc), 93-96. https://doi.org/10.1111/j.1749-6632.2009.04761.x

Hove, M. J., Marie, C., Bruce, I. C., \& Trainor, L. J. (2014). Superior time perception for lower musical pitch explains why bass-ranged instruments lay down 
musical rhythms. Proceedings of the National Academy of Sciences, 111(28), 10383-10388. https://doi.org/10.1073/pnas.1402039111

Janata, P., Tomic, S. T., \& Haberman, J. M. (2012). Sensorimotor coupling in music and the psychology of the groove. Journal of Experimental Psychology: General, 141(1), 54-75. https://doi.org/10.1037/a0024208

Kung, S.-J., Chen, J. L., Zatorre, R. J., \& Penhune, V. B. (2013). Interacting cortical and basal ganglia networks underlying finding and tapping to the musical beat. Journal of Cognitive Neuroscience, 25(3). https://doi.org/10.1162/ jocn_a_00325

Lakatos, P., Karmos, G., Mehta, A. D., Ulbert, I., \& Schroeder, C. E. (2008). Entrainment of Neuronal Oscillations as a Mechanism of Attentional Selection. Science, 320, 110-113.

Lakatos, P., Shah, A. S., Knuth, K. H., Ulbert, I., Karmos, G., \& Schroeder, C. E. (2005). An oscillatory hierarchy controlling neuronal excitability and stimulus processing in the auditory cortex. Journal of Neurophysiology, 94(3), 1904-1911.

Lancaster, J. L., Rainey, L., Summerlin, J. L., Freitas, C. S., Fox, P. T., Evans, A. C., ... Mazziotta, J. C. (1997). Automated labeling of the human brain: A preliminary report on the development and evaluation of a forward-transform method. Human Brain Mapping, 5(4), 238-242. $\quad$ https://doi.org/10.1002/(SICI)1097-0193(1997)5:4<238::AIDHBM6 $>3.0 . \mathrm{CO} ; 2-4$

Lancaster, J. L., Woldorff, M. G., Parsons, L. M., Liotti, M., Freitas, C. S., Rainey, L., ... Fox, P. T. (2000). Automated Talairach Atlas labels for functional brain mapping. Human Brain Mapping, 10(3), 120-131. https://doi.org/10.1002/1097-0193(200007)10:3<120::AID-HBM30>3.0.CO;28

Large, E. W., \& Kolen, J. F. (1994). Resonance and the percep- 
tion of musical meter. Connection Science, 6(1), 177-208. https: //doi.org/10.1080/09540099408915723

Large, E. W., \& Snyder, J. S. (2009). Pulse and Meter as Neural Resonance. Annals of the New York Academy of Sciences, 1169, 46-57. https://doi.org/10. $1111 / \mathrm{j} .1749-6632.2009 .04550 . \mathrm{x}$

Large, E. W., Herrera, J. A., \& Velasco, M. J. (2015). Neural Networks for Beat Perception in Musical Rhythm. Frontiers in Systems Neuroscience, 9(November), 159. https://doi.org/10.3389/fnsys.2015.00159

Lartillot, O., \& Toiviainen, P. (2007). A matlab toolbox for musical feature extraction from audio. International Conference on Digital Audio Effects, 1-8.

Lartillot, O., Eerola, T., Toiviainen, P., \& Fornari, J. (2008). Multi-feature modeling of pulse clarity: Design, validation, and optimization. ISMIR 2008, $521-526$.

Lerdahl, F. A., \& Jackendoff, R. S. (1983). A Generative Theory of Tonal Music. Cambridge, MA: MIT Press.

Lewis, P. A., Wing, A. M., Pope, P. A., Praamstra, P., \& Miall, R. C. (2004). Brain activity correlates differentially with increasing temporal complexity of rhythms during initialisation, synchronisation, and continuation phases of paced finger tapping. Neuropsychologia, 42(10), 1301-1312. https://doi.org/10.1016/ j.neuropsychologia.2004.03.001

Li, Z., Xiang, Q., Hockman, J., Yang, J., Yi, Y., Fujinaga, I., \& Wang, Y. (2010). A music search engine for therapeutic gait training. In Proceedings of the international conference on multimedia - mm '10 (p. 627). New York, New York, USA: ACM Press. https://doi.org/10.1145/1873951.1874037

London, J. (2012). Hearing in time: Psychological aspects of musical meter. Oxford University Press.

Longuet-Higgins, H., \& Lee, C. (1984). The rhythmic interpretation of 
monophonic music. Music Perception, 1(4), 424-440. https://doi.org/10.2307/ 40285271

Madison, G. (2006). Experiencing Groove Induced by Music: Consistency and Phenomenology. Music Perception, 24(2), 201-208.

Madison, G., \& Sioros, G. (2014). What musicians do to induce the sensation of groove in simple and complex melodies, and how listeners perceive it. Frontiers in Psychology, 5, 1-14. https://doi.org/10.3389/fpsyg.2014.00894

Madison, G., Gouyon, F., Ullén, F., \& Hörnström, K. (2011). Modeling the Tendency for Music to Induce Movement in Humans: First Correlations With Low-Level Audio Descriptors Across Music Genres. Journal of Experimental Psychology: Human Perception and Performance, 37(5), 1578-1594. https: //doi.org/10.1037/a0024323

Makeig, S., Bell, A. J., Jung, T.-P., \& Sejnowski, T. J. (1996). Independent Component Analysis of Electroencephalographic Data. In D. Touretzky, M. Mozer, \& M. Hasselmo (Eds.), Advances in neural information processing systems 8 (pp. 145-151). Cambridge, MA: MIT Press.

Makeig, S., Debener, S., Onton, J., \& Delorme, A. (2004). Mining event-related brain dynamics. Trends in Cognitive Sciences, 8(5), 204-210. https://doi.org/ 10.1016/j.tics.2004.03.008

Mayville, J. M., Fuchs, A., Ding, M., Cheyne, D., Deecke, L., \& Kelso, J. A. S. (2001). Event-related changes in neuromagnetic activity associated with syncopation and synchronization timing tasks. Human Brain Mapping, 14(2), 65-80. https://doi.org/10.1002/hbm.1042

McGarry, L. M., Russo, F. A., Schalles, M. D., \& Pineda, J. A. (2012). Audiovisual facilitation of the mu rhythm. Experimental Brain Research, 218(4), 527-538. https://doi.org/10.1007/s00221-012-3046-3

Merchant, H., Grahn, J. A., Trainor, L. J., Rohrmeier, M., \& Fitch, W. T. (2015). Finding the beat: a neural perspective across humans and non-human 
primates. Philosophical Transactions of the Royal Society of London. Series B, Biological Sciences, 370, 20140093. https://doi.org/10.1098/rstb.2014.0093

Mithen, S. (2005). The Singing Neanderthals: The Origins of Music, Language, Mind and Body. London: Weidenfeld \& Nicholson.

Nozaradan, S. (2014). Exploring how musical rhythm entrains brain activity with electroencephalogram frequency-tagging. Philosophical Transactions of the Royal Society of London. Series B, Biological Sciences, 369(1658), 1-10. https://doi.org/10.1098/rstb.2013.0393

Nozaradan, S., Peretz, I., \& Keller, P. E. (2016). Individual Differences in Rhythmic Cortical Entrainment Correlate with Predictive Behavior in Sensorimotor Synchronization. Scientific Reports, 6(20612), 1-12. https://doi.org/10. 1038/srep20612

Nozaradan, S., Peretz, I., \& Mouraux, A. (2012). Selective Neuronal Entrainment to the Beat and Meter Embedded in a Musical Rhythm. Journal of Neuroscience, 32(49), 17572-17581. https://doi.org/10.1523/JNEUROSCI.3203-12. 2012

Nozaradan, S., Peretz, I., Missal, M., \& Mouraux, A. (2011). Tagging the Neuronal Entrainment to Beat and Meter. Journal of Neuroscience, 31(28), 10234-10240. https://doi.org/10.1523/JNEUROSCI.0411-11.2011

Nozaradan, S., Zerouali, Y., Peretz, I., \& Mouraux, A. (2015). Capturing with EEG the Neural Entrainment and Coupling Underlying Sensorimotor Synchronization to the Beat. Cerebral Cortex, 25(3), 736-747. https://doi.org/10.1093/ cercor/bht261

Obleser, J., Herrmann, B., \& Henry, M. J. (2012). Neural oscillations in speech: don't be enslaved by the envelope. Frontiers in Human Neuroscience, 6(250), $1-4$.

Onton, J., Westerfield, M., Townsend, J., \& Makeig, S. (2006). Imaging human EEG dynamics using independent component analysis. Neuroscience $\mathcal{E}^{3}$ 
Biobehavioral Reviews, 30(6), 808-822.

Parncutt, R. (1994). A perceptual model of pulse salience and metrical accent in musical rhythms. Music Perception, 11(4), 409-464. https://doi.org/10.2307/ 40285633

Patel, A. D., \& Iversen, J. R. (2014). The evolutionary neuroscience of musical beat perception: the Action Simulation for Auditory Prediction (ASAP) hypothesis. Frontiers in Systems Neuroscience, 8, 1-14.

R Core Team. (2017). R: A Language and Environment for Statistical Computing (https://www.r-project.org/). Vienna, Austria: R Foundation for Statistical Computing.

Repp, B. H. (2003). Rate Limits in Sensorimotor Synchronization With Auditory and Visual Sequences: The Synchronization Threshold and the Benefits and Costs of Interval Subdivision. Journal of Motor Behavior, 35, 355-370.

Repp, B. H. (2010). Self-Generated Interval Subdivision Reduces Variability of Synchronization with a Very Slow Metronome. Music Perception, 27(5), 389397. https://doi.org/10.1525/mp.2010.27.5.389

Roholt, T. C. (2014). Groove: A phenomenology of rhythmic nuance. Bloomsbury Publishing USA.

Sioros, G., Miron, M., Davies, M., Gouyon, F., \& Madison, G. (2014). Syncopation creates the sensation of groove in synthesized music examples. Frontiers in Psychology, 5(SEP), 1-10. https://doi.org/10.3389/fpsyg.2014.01036

Smith, K. C., Cuddy, L. L., \& Upitis, R. (1994). Figural and metric understanding of rhythm. Psychology of Music, 22(2), 117-135. https://doi.org/10. $1177 / 0305735694222002$

Snyder, J. S., \& Large, E. W. (2005). Gamma-band activity reflects the metric structure of rhythmic tone sequences. Cognitive Brain Research, 24, 117-126. 
https://doi.org/10.1016/j.cogbrainres.2004.12.014

Stupacher, J., Hove, M. J., \& Janata, P. (2016). Audio features underlying perceived groove and sensorimotor synchronization in music. Music Perception, $33(5), 571-589$.

Stupacher, J., Hove, M. J., Novembre, G., Schütz-Bosbach, S., \& Keller, P. E. (2013). Musical groove modulates motor cortex excitability: A TMS investigation. Brain and Cognition, 82(2), 127-136. https://doi.org/10.1016/j.bandc. 2013.03.003

Stupacher, J., Wood, G., \& Witte, M. (2017). Neural entrainment to polyrhythms: A comparison of musicians and non-musicians. Frontiers in Neuroscience, 11(208), 1-17. https://doi.org/10.3389/fnins.2017.00208

Thaut, M. H., \& Abiru, M. (2010). Rhythmic Auditory Stimulation in Rehabilitation of Movement Disorders: A Review Of Current Research. Music Perception, 27(4), 263-269. https://doi.org/10.1525/MP.2010.27.4.263

Tierney, A. T., \& Kraus, N. (2014). Neural Entrainment to the Rhythmic Structure of Music. Journal of Cognitive Neuroscience, 27(2), 400-408. https: //doi.org/10.1162/jocn_a_00704

Toiviainen, P., \& Eerola, T. (2016). MIDI toolbox 1.1. https://github.com/miditoolbox/; GitHub.

Velasco, M. J., \& Large, E. W. (2011). Pulse Detection in Syncopated Rhythms Using Neural Oscillators. 12th International Society for Music Information Retreival Converence, 185-190.

Vuust, P., \& Witek, M. A. G. (2014). Rhythmic complexity and predictive coding: A novel approach to modeling rhythm and meter perception in music. Frontiers in Psychology, 5(OCT), 1-14. https://doi.org/10.3389/fpsyg.2014.01111.

Vuust, P., Gebauer, L. K., \& Witek, M. A. G. (2014). Neural Underpinnings of Music: The Polyrhythmic Brain. In Neurobiology of interval timing (pp. 
339-356). https://doi.org/10.1007/978-1-4939-1782-2

Winkler, I., Debener, S., Muller, K. R., \& Tangermann, M. (2015). On the influence of high-pass filtering on ICA-based artifact reduction in EEG-ERP. Proceedings of the Annual International Conference of the IEEE Engineering in Medicine and Biology Society, EMBS, 2015-Novem, 4101-4105. https://doi. org/10.1109/EMBC.2015.7319296

Witek, M. A. G. (2017). Filling In: Syncopation, Pleasure and Distributed Embodiment in Groove. Music Analysis, 36(1), 138-160. https://doi.org/10. 1111/musa. 12082

Witek, M. A. G., Clarke, E. F., Wallentin, M., Kringelbach, M. L., \& Vuust, P. (2014). Syncopation, Body-Movement and Pleasure in Groove Music. PLoS ONE, 9(4). https://doi.org/10.1371/journal.pone.0094446

Witek, M. A. G., Popescu, T., Clarke, E. F., Hansen, M., Konvalinka, I., Kringelbach, M. L., \& Vuust, P. (2017). Syncopation affects free body-movement in musical groove. Experimental Brain Research, 235(4), 995-1005. https: //doi.org/10.1007/s00221-016-4855-6

Yin, S., Liu, Y., \& Ding, M. (2016). Amplitude of Sensorimotor Mu Rhythm Is Correlated with BOLD from Multiple Brain Regions: A Simultaneous EEGfMRI Study. Frontiers in Human Neuroscience, 10(July), 1-12. https://doi. org/10.3389/fnhum.2016.00364

Zanto, T. P., Snyder, J. S., \& Large, E. W. (2006). Neural correlates of rhythmic expectancy. Advances in Cognitive Psychology, 2(2-3), 221-231.

Zatorre, R. J., Chen, J. L., \& Penhune, V. B. (2007). When the brain plays music: auditory-motor interactions in music perception and production. Nature Reviews Neuroscience, 8(7), 547-558. https://doi.org/10.1038/nrn2152 